\title{
MULTIPLE CONDENSATIONS FOR A NONLINEAR ELLIPTIC EQUATION WITH SUB-CRITICAL GROWTH AND CRITICAL BEHAVIOUR
}

\author{
JUNCHENG WEI \\ Department of Mathematics, Chinese University of Hong Kong, \\ Shatin, Hong Kong (wei@math.cuhk.edu.hk)
}

(Received 26 April 1999)

Abstract We consider the following nonlinear elliptic equations

$$
\begin{cases}\Delta u+u_{+}^{N /(N-2)}=0 & \text { in } \Omega \\ u=\mu & \text { on } \partial \Omega \quad(\mu \text { is an unknown constant }) \\ \int_{\partial \Omega}\left(-\frac{\partial u}{\partial n}\right)=M, & \end{cases}
$$

where $u_{+}=\max (u, 0), M$ is a prescribed constant, and $\Omega$ is a bounded and smooth domain in $R^{N}$, $N \geqslant 3$. It is known that for $M=M_{*}^{(N)}, \Omega=B_{R}(0)$, the above problem has a continuum of solutions. The case when $M>M_{*}^{(N)}$ is referred to as supercritical in the literature. We show that for $M$ near $K M_{*}^{(N)}, K>1$, there exist solutions with multiple condensations in $\Omega$. These concentration points are non-degenerate critical points of a function related to the Green's function.

Keywords: nonlinear elliptic equations; free boundary; singular perturbations; critical behaviour

AMS 2000 Mathematics subject classification: Primary 35B40; 35B45

Secondary $35 \mathrm{~J} 40$

\section{Introduction}

Let $\Omega$ be a bounded domain in $R^{N}$ with a smooth boundary. Consider the following nonlinear boundary-value problem,

$$
\begin{cases}\Delta u+g(u, x)=0 & \text { on } \Omega \\ u=\mu & \text { on } \partial \Omega \quad(\mu \text { is an unknown constant }), \\ \int_{\partial \Omega}\left(-\frac{\partial u}{\partial n}\right)=M, & \end{cases}
$$

where $g(\cdot, \cdot) \geqslant 0$ on $R^{1} \times \Omega$, and $M>0$ is a prescribed constant.

Equation (1.1) arises in several applications. In particular, if $g(t, x)=K(x) \mathrm{e}^{t}, N=2$, the solutions to problem (1.1) yield Riemannian metrics that are conformally equivalent to the Euclidean metrics on $\Omega$ with a given Gaussian curvature $K$ and a prescribed total 
curvature $M / 2[\mathbf{2 1}, \mathbf{3 9}]$. If $g(t, x)=f(t-V(x))$, where $V(x) \in C^{0}(\bar{\Omega})$ is given and $f(\cdot)$ is non-decreasing, then the solutions of (1.1) represent potentials of stationary distributions of self-gravitating clusters. $M$ stands for the total mass of the cluster (see $[\mathbf{3 8}]$ ).

In this paper, we study a particular type of (1.1), namely,

$$
\left.\begin{array}{c}
-\Delta u=u_{+}^{p}, \\
u=\mu \quad \text { on } \partial \Omega \quad(\mu \text { is an unknown constant }), \\
-\int_{\partial \Omega} \frac{\partial u}{\partial n}=M,
\end{array}\right\}
$$

where $N \geqslant 3,1<p<2^{*}:=(N+2) /(N-2)$ and $u_{+}=\max (u, 0)$.

The existence of solutions to (1.2) in the case of $1 \leqslant p<N /(N-2)=p^{*}$ was first established by Berestycki and Brezis $[\mathbf{8}]$ (Temam [31] studied the case $p=1$ ). Wang $[\mathbf{3 2}]$ extended this result to $p^{*} \leqslant p<2^{*}=(N+2) /(N-2)$ (for $M$ small). Bandle and Marcus [4] studied a priori estimates for (1.2) under assumptions similar to those in [8]. For more general $g$, see $[\mathbf{3 9}]$.

The exponent $p^{*}=N /(N-2)$ turns out to be a natural critical exponent for (1.2). Even though it has subcritical growth, it has critical behaviour. Wolansky [39] introduced an energy functional to (1.2). It turns out that when $M>M_{*}^{(N)}$, the functional becomes unbounded and non-compact and therefore it has loss of compactness similar to nonlinear elliptic equations with critical Sobolev exponent $2^{*}$, as studied in $[\mathbf{1}, \mathbf{2}, \mathbf{5}, \mathbf{1 1}, \mathbf{2 9}, \mathbf{3 0}, \mathbf{3 5}$, among others. When $\Omega=B_{1}(0)$, one can find a continuum of solutions to (1.2). In fact, let $\psi(r)$ be the solution of

$$
\begin{gathered}
\frac{\partial^{2}}{\partial r^{2}} \psi+\frac{N-1}{r} \frac{\partial}{\partial r} \psi+\left(\psi_{+}\right)^{p^{*}}=0, \\
\frac{\partial \psi}{\partial r}(0)=0, \quad \psi(0)=1 .
\end{gathered}
$$

Let $r_{0}>0$ be the smallest root such that $\psi\left(r_{0}\right)=0$. Set

$$
M_{*}^{(N)}:=\omega_{N} \int_{0}^{r_{0}} s^{N-1} \psi^{p^{*}}(s) \mathrm{d} s,
$$

where $\omega_{N}$ stands for the area of the unit sphere $S^{N-1} \subset R^{N}$.

It can easily be shown that for any $\alpha \geqslant 1$, the function

$$
U^{\alpha}(r)= \begin{cases}\alpha^{N-2} r_{0}^{N-2} \psi\left(\alpha r_{0} r\right), & \text { for } 0 \leqslant r \leqslant \alpha^{-1}, \\ \omega_{N}^{-1} M_{*}^{(N)}\left(r^{2-N}-1\right)-\omega_{N}^{-1} M_{*}^{(N)}\left(\alpha^{N-2}-1\right), & \text { for } \alpha^{-1} \leqslant r \leqslant 1,\end{cases}
$$

is a solution of (1.2) for $p=p^{*}, M=M_{*}^{(N)}$.

In the general domain, the existence of solutions of (1.2) is related to the Green's function in $\Omega$. Let $G(x, y)$ be the Green's function of $-\Delta$ in $H_{0}^{1}(\Omega)$, and let $\Gamma(|x-y|)$ be the singular part of $G(x, y)$, i.e.

$$
\Gamma(|x-y|)=\frac{1}{(N-2)\left|S^{N-1}\right||x-y|^{N-2}},
$$


where $S^{N-1}$ is the unit sphere in $R^{N}$. The regular part of $G(x, y)$ is defined as $H(x, y)=$ $\Gamma(|x-y|)-G(x, y)$. (The function $H(x, x)$ is called the Robin function. For more properties of the Robin function, see $[\mathbf{3}]$.)

Wolansky [39] proved the following theorem.

Theorem A. Assume that there exists $x_{0} \in \Omega$ for which

$$
H\left(x_{0}, y\right)>H\left(x_{0}, x_{0}\right), \quad \forall y \in \Omega \backslash\left\{x_{0}\right\} .
$$

Then, for any $0<M<M_{*}^{(N)}$ (defined by (1.4)) there exists a solution $u^{M}$ of (1.2) for $p=p^{*}$. Moreover, as $M \rightarrow M_{*}^{(N)}, \mu^{M} \rightarrow-\infty$.

A natural question is what happens when $M>M_{*}^{(N)}$ ? As far as we know, there are no existence results yet. The purpose of this paper is to answer this question.

Fix $\boldsymbol{P}=\left(P^{1}, \ldots, P^{K}\right) \in \Omega^{K}:=\Omega \times \Omega \times \cdots \times \Omega$. Set

$$
F(\boldsymbol{P})=-\sum_{j=1}^{K} H\left(P^{j}, P^{j}\right)+\sum_{l, m, l \neq m} G\left(P^{l}, P^{m}\right) .
$$

We then have the following theorem.

Theorem 1.1. Let $K \geqslant 1, K \in \mathcal{N}$. Assume that $\boldsymbol{P}_{0}=\left(P_{0}^{1}, \ldots, P_{0}^{K}\right) \in \Omega^{K}$ is a nondegenerate critical point of $F(\boldsymbol{P})$. Let $p=p^{*}$. Then there exists an $\epsilon_{0}>0$ such that for every $\epsilon \in\left(0, \epsilon_{0}\right)$, there exists a solution $u^{M}$ to (1.2) satisfying the following properties.

(i) $\left.u^{M}\right|_{\partial \Omega}=\mu^{M}=-\epsilon^{-2 /(p-1)} \rightarrow-\infty$,

(ii) The set $\left\{u^{M}>0\right\}$ contains exactly $K$ connected domains.

(iii) $M=K M_{*}^{(N)}+o\left(\left(-\mu^{M}\right)^{-1}\right)$ is a continuous function of $\epsilon \in\left(0, \epsilon_{0}\right)$.

Remark 1.2. A more detailed description of the free boundary $\left\{u^{M}>0\right\}$ can be found in Theorem 1.4 below.

In fact, for any $1<p<2^{*}, p \neq p^{*}$, we have the following theorem.

Theorem 1.3. Assume that $\boldsymbol{P}_{0}=\left(P_{0}^{1}, \ldots, P_{0}^{K}\right) \in \Omega^{K}$ is a non-degenerate critical point of $F(\boldsymbol{P})$. Let $1<p<2^{*}, p \neq p^{*}$. Then there exist $\delta_{0}, C_{0}>0$ such that for every $M \in\left(0, \delta_{0}\right)$ if $p^{*}<p<2^{*}$ and $M \in\left(C_{0},+\infty\right)$ if $1<p<p^{*}$, there exists a solution $u^{M}$ to (1.2) satisfying the following properties, as $M \rightarrow 0$ for $p>p^{*}$ or $M \rightarrow+\infty$ for $p<p^{*}$.

(i) The set $\left\{u^{M}>0\right\}$ contains exactly $K$ connected domains.

(ii) $\left.u^{M}\right|_{\partial \Omega}=\mu^{M} \rightarrow-\infty$.

(iii) $M\left(-\mu^{M}\right)^{(2 p-N(p-1)) / 2} \rightarrow \mu_{0}$, where $\mu_{0}$ is a generic constant. 
Let us now introduce the main idea of the proofs. One of the key points is the following transformation of (1.2): let $\mu=-\epsilon^{-2 /(p-1)}$ and $u=|\mu| v+\mu$, then we have that equation (1.2) is equivalent to finding $(v, \epsilon)$ such that

$$
\begin{cases}\epsilon^{2} \Delta v+(v-1)_{+}^{p^{*}}=0, & v>0 \text { in } \Omega, \\ v=0, & \text { on } \partial \Omega, \\ \epsilon^{-2 p /(p-1)} \int_{\Omega}(v-1)_{+}^{p}=M . & \end{cases}
$$

Note that in problem (1.8), $M$ is fixed and $\epsilon>0$ is undetermined. To solve (1.8), we first solve the following elliptic equation

$$
\begin{cases}\epsilon^{2} \Delta v+(v-1)_{+}^{p}=0 & \text { in } \Omega, \\ v>0, & \text { in } \Omega, \\ v=0, & \text { on } \partial \Omega,\end{cases}
$$

for $\epsilon$ small, and then we solve the following algebraic equation

$$
M=\epsilon^{-2 p /(p-1)} \int_{\Omega}(v-1)_{+}^{p} .
$$

Equation (1.9) can be regarded as a singular perturbation problem with a free boundary. The boundary of the core

$$
A:=\{v>1\}
$$

is the free boundary for (1.9). Problem (1.9) arises in plasma physics $[\mathbf{3}, \mathbf{1 2}, \mathbf{2 0}]$. The corresponding problem in two dimensions has been studied in $[\mathbf{3}]$; the case when $p=1$ by Caffarelli and Friedman [12]. The higher-dimensional case has been studied by Flucher and the author in [20]. For more information and background for (1.9), please see [20] and the references cited therein. It was proved in [20] that as $\epsilon \rightarrow 0$ the least-energy solution has one single local maximum $x_{\epsilon} \in \Omega$ and $H\left(x_{\epsilon}, x_{\epsilon}\right) \rightarrow \min _{x \in \Omega} H(x, x)$. In this paper, we shall study solutions with multiple condensations.

Our main result for (1.9) is the following theorem.

Theorem 1.4. Let $1<p<(N+2) /(N-2)_{+}, N \geqslant 3$, and suppose that $\boldsymbol{P}_{0}$ is a non-degenerate critical point of $F(\boldsymbol{P})$. Then for $\epsilon \ll 1$, problem (1.9) admits a solution $u_{\epsilon}$ such that $u_{\epsilon}$ has only $K$ local maximum points $P_{\epsilon}^{j}, j=1, \ldots, K$, and $P_{\epsilon}^{j} \rightarrow P_{0}^{j}$, $j=1, \ldots, K$, as $\epsilon \rightarrow 0$. The core $A_{\epsilon}:=\left\{u_{\epsilon}>1\right\}$ has exactly $K$ connected components and

$$
\bigcup_{j=1}^{K} B_{R_{2} \epsilon}\left(P_{\epsilon}^{j}\right) \subset A_{\epsilon} \subset \bigcup_{j=1}^{K} B_{R_{1} \epsilon}\left(P_{\epsilon}^{j}\right)
$$

for some numbers $R_{1}>R_{2}>0, R_{1}=R_{w}+o(1), R_{2}=R_{w}+o(1)$, where $R_{w}$ is a generic constant defined in $\S 2$.

Remark 1.5. When $K=1$, Theorem 1.4 can be considered as a converse of Theorem 4 in [20]. 
To prove Theorems 1.1 and 1.3, we need the following theorem.

Theorem 1.6. Let $\boldsymbol{P}_{0}$ be a non-degenerate critical point of $F(\boldsymbol{P})$ and $u_{\epsilon}$ be the solution constructed by Theorem 1.4. Then, for $\epsilon$ small, $u_{\epsilon}$ is unique and we have

$$
I(\epsilon):=\epsilon^{-2 p /(p-1)} \int_{\Omega}\left(u_{\epsilon}-1\right)_{+}^{p}=K I_{p}+d_{w} F\left(\boldsymbol{P}^{0}\right) \epsilon^{N-2}+o\left(\epsilon^{N-2}\right) .
$$

is a continuous function of $\epsilon$, where $I_{p}>0, d_{w}$ are defined in $\S 5$. In particular, when $p=p^{*}, I_{p}=M_{*}^{(N)}, d_{w}=0$.

Theorems 1.1 and 1.3 follow directly from Theorem 1.6.

Remark 1.7. It is interesting to note that results similar to Theorem 1.4 appear frequently in the study of problems arising from applied sciences and geometry. It turns out that Green's function is naturally associated with such phenomenon. For blow-up problems involving the Sobolev critical exponent, see $[\mathbf{1}, \mathbf{2}, \mathbf{1 1}, \mathbf{2 9}, \mathbf{3 0}, \mathbf{3 5}]$ and the references cited therein. For multiple concentrations in singular perturbation problems, see $[\mathbf{6}, \mathbf{7}, \mathbf{1 5}-\mathbf{1 9}, \mathbf{2 2}, \mathbf{2 3}, \mathbf{2 7}, \mathbf{3 7}]$ and the references cited therein. Our problem (1.9) can be considered as a borderline case of singular perturbation problems. Most of the references cited here deal with singular perturbation problems of the kind $\epsilon^{2} \Delta u+f(u)=0$, $f^{\prime}(0)<0$. In our case, we have $f^{\prime}(0)=0$, which is called the zero-mass case $[\mathbf{9}]$. In the zero-mass case, the decay of the ground state is of algebraic order and therefore the traditional Sobolev space $H^{2}$ does not work. Here we use a weighted Sobolev space approach.

Remark 1.8. We remark that our results here in some sense are a generalization of those in [5]. In [5], Baraket and Pacard considered the following problem:

$$
\begin{cases}\Delta u+\rho \mathrm{e}^{u}=0, & \text { in } \Omega, \\ u=0, & \text { on } \partial \Omega,\end{cases}
$$

where $\Omega$ is a smooth bounded domain in $R^{2}$.

They introduced a function $\mathcal{F}(\boldsymbol{P})$ in $R^{2}$ that is equal to our $F(\boldsymbol{P})$ in $R^{2}$. Then they proved that at any non-degenerate critical point $\boldsymbol{P}_{0}=\left(P_{0}^{1}, \ldots, P_{0}^{K}\right)$ of $F(\boldsymbol{P})$, for $\rho$ small, there exists a solution $u_{\rho}$ that blows up near $P_{0}^{j}, j=1, \ldots, K$. Moreover, it can be shown that $\rho \int_{\Omega} \mathrm{e}^{u_{\rho}} \rightarrow 8 \pi K$. In some sense they established the results similar to our Theorem 1.4 in the case $N=2$. There they used a weighted Hölder space approach. However, they did not show that the solution $u_{\rho}$ is unique (though uniqueness is very likely to be true), and thus they do not have results similar to our Theorem 1.1.

Remark 1.9. The non-degeneracy condition in Theorem 1.4 may be relaxed a little bit. For example, if we have strictly local maximum point $\boldsymbol{P}_{0}$ or strictly local minimum point of $F(\boldsymbol{P})$, we can use the method of minimization or maximization of the reduced energy (similar to $[\mathbf{1 8}]$ or $[\mathbf{1 9}]$ ) to obtain solutions of (1.9) with multiple condensations. It seems hard to remove the non-degeneracy condition in Theorems 1.1 and 1.3. The main difficulty is in showing that $I(\epsilon)$ is continuous in $\epsilon$. 
Remark 1.10. An interesting question is whether there exists a solution to (1.8) when $M=K M_{*}^{(N)}$. We learned from Chang that she and Lin have obtained some results for this question in the case when $N=2$ [14]. The higher-dimensional case is still open.

Remark 1.11. Although we have just studied a particular type of problem (1.1), the results in this paper can certainly be generalized to deal with problem (1.1) with $g(u, x)=f(u-V(x))$ for some $f$ and $V(x) \in C^{2}$. We shall not pursue this generality here.

The organization of the paper is as follows. In $\S 2$, we first introduce a projection and study the properties of the projection. Then we introduce some Sobolev spaces and study the properties of the linearized operator in these spaces. Section 3 contains the classical Liapunov-Schmidt reduction process. Here we reduce our problem to a finite-dimensional one. In $\S 4$, we solve the finite-dimensional problem and prove Theorem 1.4. In $\S 5$, we show that the solutions constructed in $\S 4$ are unique. In $\S 6$, we return to the study of (1.2) and prove Theorems 1.1 and 1.3. Finally we prove some technical lemmas in $\S \S 7$ and 8 .

Throughout this paper, the letters $C, c, C_{i}$ and $c_{i}$ will denote various constants independent of $\epsilon$ small. $\delta$ will always denote a small constant.

\section{Preliminaries}

In this section we introduce some notation and prove necessary estimates.

Let $f(u):=(u-1)_{+}^{p}$ for $1<p<(N+2) /(N-2)$. It is known that the following problem in $R^{N}$,

$$
\begin{cases}\Delta w+(w-1)_{+}^{p}=0, & \text { in } R^{N}, \\ w>0, & w(0)=\max _{y \in R^{N}} w(y), \\ w(y) \rightarrow 0, & \text { as }|y| \rightarrow+\infty,\end{cases}
$$

has a unique 'ground-state' solution $w$. Moreover, we have the following lemma.

Lemma 2.1 (see $\S 4$ in [20]). $w=w(r), w^{\prime}(r)<0$ for $r \neq 0$ and

$$
w(r)=\frac{k_{w}}{(N-2)\left|S^{N-1}\right|} r^{2-N}
$$

for $r \geqslant R_{w}$, where $k_{w}>0, R_{w}>0$.

Next fix any $P \in \Omega$. We project ' $w$ ' into $H_{0}^{1}(\Omega)$ by defining $w_{\epsilon, P}$ to be the unique solution of

$$
\begin{cases}\epsilon^{2} \Delta w_{\epsilon, P}+\left(w\left(\frac{x-P}{\epsilon}\right)-1\right)_{+}^{p}=0, & \text { in } \Omega, \\ w_{\epsilon, P}=0, & \text { on } \partial \Omega .\end{cases}
$$

Note that $w_{\epsilon, P}$ is $C^{2}$ in $P$. Then we have the following lemma by Lemma 2.1. 


\section{Lemma 2.2.}

$$
w_{\epsilon, P}(x)=w\left(\frac{x-P}{\epsilon}\right)-\epsilon^{N-2} k_{w} H(x, P), \quad \text { if } d(P, \partial \Omega)>R_{w} \epsilon,
$$

and

$$
w_{\epsilon, P}(x)=k_{w} \epsilon^{N-2} G(x, P), \quad \text { for }|x-P|>R_{w} \epsilon, \quad d(P, \partial \Omega)>R_{w} \epsilon .
$$

Let $\boldsymbol{P}=\left(P^{1}, \ldots, P^{K}\right) \in \Omega^{K}$ and $\boldsymbol{P}_{0}$ be a critical point of $F(\boldsymbol{P})$. From now on, we will always assume that

$$
\boldsymbol{P} \in B_{\delta}\left(\boldsymbol{P}_{0}\right)
$$

for some $\delta>0$ small.

We denote $P_{i}^{j}$ to be the $i$ th component of $P^{j}, j=1, \ldots, K$.

Define

$$
\begin{gathered}
w_{j}(x)=w\left(\frac{x-P^{j}}{\epsilon}\right), \quad w_{\epsilon, \boldsymbol{P}}=\sum_{j=1}^{K} w_{\epsilon, P^{j}}, \\
\partial_{i}^{j}=\frac{\partial}{\partial P_{i}^{j}}, \quad j=1, \ldots, K, \quad i=1, \ldots, N, \\
\Omega_{\epsilon, P^{j}}:=\left\{y \mid \epsilon y+P^{j} \in \Omega\right\} .
\end{gathered}
$$

Set

$$
F_{j}(\boldsymbol{P}, x)=\sum_{l \neq j} G\left(x, P^{l}\right)-H\left(x, P^{j}\right) .
$$

Then it is easy to see that

$$
F(\boldsymbol{P})=\sum_{j=1}^{K} F_{j}\left(\boldsymbol{P}, P^{j}\right) .
$$

We have the following error estimates.

Lemma 2.3. For $\epsilon$ sufficiently small, we have

$$
\operatorname{support}\left(\epsilon^{2} \Delta w_{\epsilon, \boldsymbol{P}}+\left(w_{\epsilon, \boldsymbol{P}}-1\right)_{+}^{p}\right) \subset \bigcup_{j=1}^{K} B_{R_{0} \epsilon}\left(P^{j}\right),
$$

where $R_{0}=R_{w}+o(1)>0$. Moreover,

$$
\epsilon^{2} \Delta w_{\epsilon, \boldsymbol{P}}+\left(w_{\epsilon, \boldsymbol{P}}-1\right)_{+}^{p}=p(w-1)_{+}^{p-1} \epsilon^{N-2} k_{w} F_{j}\left(\boldsymbol{P}, P^{j}\right)+O\left(\epsilon^{N-1}\right),
$$

for $x \in B_{R_{0} \epsilon}\left(P^{j}\right)$, and

$$
\begin{aligned}
& \epsilon^{2} \Delta w_{\epsilon, \boldsymbol{P}}(x)+\left(w_{\epsilon, \boldsymbol{P}}-1\right)_{+}^{p}(x) \\
& =f_{\epsilon, P^{j}}(|y|)+p(w(y)-1)_{+}^{p-1} k_{w} \\
& \quad \times\left.\sum_{k=1}^{N} \frac{\partial}{\partial x_{k}} F_{j}(\boldsymbol{P}, x)\right|_{x=P^{j}} \epsilon^{N-1} y_{k}+O\left(\epsilon^{N}\right), \quad x \in B_{R_{0} \epsilon}\left(P^{j}\right),
\end{aligned}
$$

where $\epsilon y+P^{j}=x, f_{\epsilon, P^{j}}$ is a radial function and is supported in $B_{R_{0}}(0)$. 
Proof. Observe that by Lemma 2.2, for $x \in B_{2 R_{w} \epsilon}\left(P^{j}\right)$,

$$
w_{\epsilon, \boldsymbol{P}}(x)=\sum_{j=1}^{K} w_{\epsilon, P^{j}}(x)=w\left(\frac{x-P^{j}}{\epsilon}\right)+\epsilon^{N-2} k_{w} F_{j}(\boldsymbol{P}, x) .
$$

Equations (2.6) and (2.7) follow from direct computations.

Next we introduce some Sobolev spaces.

We first define weighted Sobolev space in $R^{N}$. For $1<t<\infty$, a non-negative integer $l$ and a real number $\beta$, the weighted Sobolev space $W_{l, \beta}^{t}\left(R^{N}\right)$ is defined to be the completion of $C_{0}^{\infty}\left(R^{N}\right)$ under the norm:

$$
\sum_{|\alpha|=0}^{l}\left\|\langle x\rangle^{\beta+|\alpha|} \partial^{\alpha} u\right\|_{L^{t}\left(R^{N}\right)},
$$

where $\langle x\rangle=\left(1+|x|^{2}\right)^{1 / 2}$. If $\beta=0=l$, then $W_{l, \beta}^{t}\left(R^{N}\right)$ is just the usual $L^{t}\left(R^{N}\right)$ space. When $l=0$, we write $W_{0, \beta}^{t}\left(R^{N}\right)$ as $L_{\beta}^{t}\left(R^{N}\right)$. The properties of these Sobolev spaces can be found in $[\mathbf{1 3}, \mathbf{2 4}, \mathbf{2 5}, \mathbf{2 8}]$.

For any bounded open set $U$, we can define $W_{l, \beta}^{t}(U)$ to be the space $W^{l, t}(U)$ equipped with the norm:

$$
\sum_{|\alpha|=0}^{l}\left\|\langle x\rangle^{\beta+|\alpha|} \partial^{\alpha} u\right\|_{L^{t}(U)} .
$$

Similarly we can define $L_{\beta}^{t}(U)$.

Let $t^{\prime}$ denote, as always, the conjugate of $t$ :

$$
\frac{1}{t}+\frac{1}{t^{\prime}}=1
$$

We now turn to the linear operator

$$
L:=\Delta+p(w-1)_{+}^{p-1}: W_{2, \beta}^{t}\left(R^{N}\right) \rightarrow L_{\beta+2}^{t}\left(R^{N}\right) .
$$

We then have the following lemma.

Lemma 2.4. If

$$
1-\frac{N}{t}<\beta<\frac{N}{t^{\prime}}-1
$$

then

$$
\operatorname{Kernel}(L) \cap W_{2, \beta}^{t}\left(R^{N}\right)=X_{0}:=\operatorname{span}\left\{\frac{\partial w}{\partial y_{i}} \mid i=1, \ldots, N\right\} .
$$

Proof. By direct computations,

$$
\left|\frac{\partial w}{\partial y_{i}}\right| \leqslant C|y|^{1-N}
$$

for $|y|>R_{w}$. Hence $X_{0} \subset \operatorname{kernel}(L) \cap W_{2, \beta}^{t}\left(R^{N}\right)$, since $\beta<\left(N / t^{\prime}\right)-1$. The rest follows from the same proof of Proposition 2.1 in [33], Lemma 4.2 in [32], or Lemma 6 in [20]. 
Observe that when $\beta>1-(N / t), u v \in L^{1}\left(R^{N}\right)$ if $u \in L_{\beta}^{t}, v \in X_{0}$. Hence if $1-(N / t)<$ $\beta<\left(N / t^{\prime}\right)-1$, we can decompose $W_{2, \beta}^{t}\left(R^{N}\right)$ as follows

$$
W_{2, \beta}^{t}\left(R^{N}\right)=X_{0} \oplus X_{0}^{\perp},
$$

where

$$
X_{0}^{\perp}=\left\{u \in W_{2, \beta}^{t}\left(R^{N}\right) \mid \int_{R^{N}} u v \mathrm{~d} x=0, \forall v \in X_{0}\right\} .
$$

Let us denote

$$
Y_{0}:=\left\{\frac{\partial w}{\partial y_{i}}, i=1, \ldots, N\right\} \subset L_{\beta+2}^{t}\left(R^{N}\right)
$$

and

$$
Y_{0}^{\perp}=\left\{u \in L_{2+\beta}^{t}\left(R^{N}\right) \mid \int_{R^{N}} u v=0, \forall v \in Y_{0}\right\} .
$$

Then we have the following proposition.

Proposition 2.5. Suppose that $N \geqslant 3$ and $1-(N / t)<\beta<\left(N / t^{\prime}\right)-1$, then

$$
L^{-1}: Y_{0}^{\perp} \rightarrow X_{0}^{\perp}
$$

exists and is bounded.

Proof. See Proposition 2.3 in [33].

Proposition 2.5 will have a natural analogue in $\Omega$. We shall discuss this in the rest of this section. Before that, we introduce some notation and some technical lemmas.

Set

$$
X_{\epsilon, \boldsymbol{P}}^{t, \beta}=\cap_{j=1}^{K} W_{2, \beta}^{t}\left(\Omega_{\epsilon, P^{j}}\right) \cap W_{0}^{1,2}(\Omega),
$$

and

$$
Y_{\epsilon, \boldsymbol{P}}^{t, \beta}=\cap_{j=1}^{K} L_{\beta}^{t}\left(\Omega_{\epsilon, P^{j}}\right)
$$

In $X_{\epsilon, \boldsymbol{P}}^{t, \beta}$, we define its norm as

$$
\|u\|_{t, 2, \beta}=\sum_{|\alpha|=0}^{2}\left(\epsilon^{-N} \int_{\Omega}\left|\left(\sigma_{\boldsymbol{P}}(x)\right)^{\beta+|\alpha|} \partial^{\alpha} u\right|^{t}\right)^{1 / t},
$$

where

$$
\sigma_{P}(x):=\min _{j=1}^{K}\left(1+\left|\frac{x-P^{j}}{\epsilon}\right|^{2}\right)^{1 / 2}
$$


Similarly in $Y_{\epsilon, \boldsymbol{P}}^{t, 2+\beta}$ we define the norm as

$$
\|u\|_{t, 0,2+\beta}=\left(\epsilon^{-N} \int_{\Omega}\left|\left(\sigma_{\boldsymbol{P}}(x)\right)^{2+\beta} u\right|^{t}\right)^{1 / t} .
$$

From now on, we drop the indexes $\epsilon, \boldsymbol{P}$ in $X_{\epsilon, \boldsymbol{P}}^{t, \beta}, Y_{\epsilon, \boldsymbol{P}}^{t, 2+\beta}$ if there is no confusion.

We then have the following technical lemma whose proof will be given in Appendix B.

Lemma 2.6. Suppose that $0<\beta<\left(N / t^{\prime}\right)-1$. Let $\Phi$ satisfy the following equation:

$$
\begin{cases}\epsilon^{2} \Delta \Phi+f=0, & \text { in } \Omega, \\ \Phi=0, & \text { on } \partial \Omega .\end{cases}
$$

Then we have

$$
\|\Phi\|_{t, 2, \beta} \leqslant C\|f\|_{t, 0,2+\beta} .
$$

Remark 2.7. If $K=1$, we just need $1-(N / t)<\beta<\left(N / t^{\prime}\right)-1$. If $K>1, \beta>0$ is needed for technical reasons.

Set

$$
L_{\epsilon, \boldsymbol{P}}=\epsilon^{2} \Delta+p\left(w_{\epsilon, \boldsymbol{P}}-1\right)_{+}^{p-1} .
$$

From now on, we restrict the exponents $t, \beta$ such that

$$
\frac{N}{N-1}<t<N, \quad 0<\beta<\frac{N}{t^{\prime}}-1 .
$$

We now study the linear operator $L_{\epsilon, \boldsymbol{P}}$ in $X^{t, \beta}$ and prove an analogue of Proposition 2.5 .

Set

$$
\begin{aligned}
& \mathcal{K}_{\epsilon, \boldsymbol{P}}=\operatorname{span}\left\{\partial_{i}^{j} w_{\epsilon, \boldsymbol{P}} \mid 1 \leqslant j \leqslant K, 1 \leqslant i \leqslant N\right\} \subset X^{t, \beta}, \\
& \mathcal{C}_{\epsilon, \boldsymbol{P}}=\operatorname{span}\left\{\partial_{i}^{j} w_{\epsilon, \boldsymbol{P}} \mid 1 \leqslant j \leqslant K, 1 \leqslant i \leqslant N\right\} \subset Y^{t, 2+\beta}, \\
& \mathcal{K}_{\epsilon, \boldsymbol{P}}^{\perp}=\left\{u \in X^{t, \beta} \mid \int_{\Omega} u \partial_{i}^{j} w_{\epsilon, \boldsymbol{P}}=0,1 \leqslant j \leqslant K, 1 \leqslant i \leqslant N\right\}, \\
& \mathcal{C}_{\epsilon, \boldsymbol{P}}^{\perp}=\left\{u \in Y^{t, 2+\beta} \mid \int_{\Omega} u \partial_{i}^{j} w_{\epsilon, \boldsymbol{P}}=0,1 \leqslant j \leqslant K, 1 \leqslant i \leqslant N\right\} .
\end{aligned}
$$

Let $\Pi_{\epsilon, \boldsymbol{P}}$ and $\Pi_{\epsilon, \boldsymbol{P}}^{\perp}$ be the projections of $Y^{t, 2+\beta}$ into $\mathcal{C}_{\epsilon, \boldsymbol{P}}$ and $\mathcal{C}_{\epsilon, \boldsymbol{P}}^{\perp}$, respectively.

The following is the main estimate we need in order to apply the Liapunov-Schmidt reduction method.

Proposition 2.8. Let $0<\beta<\left(N / t^{\prime}\right)-1$ and $(N /(N-1))<t<N$. Then for $\epsilon \ll 1$ and $\boldsymbol{P} \in B_{\delta}\left(\boldsymbol{P}_{0}\right)$, the operator defined by

$$
\tilde{L}_{\epsilon, \boldsymbol{P}}:=\Pi_{\epsilon, \boldsymbol{P}}^{\perp} \circ L_{\epsilon, \boldsymbol{P}}: \mathcal{K}_{\epsilon, \boldsymbol{P}}^{\perp} \rightarrow \mathcal{C}_{\epsilon, \boldsymbol{P}}^{\perp}
$$

is one-to-one and surjective (and hence invertible). Moreover,

$$
\left\|\tilde{L}_{\epsilon, \boldsymbol{P}} \Phi\right\|_{t, 0,2+\beta} \geqslant C\|\Phi\|_{t, 2, \beta}, \quad \forall \Phi \in \mathcal{K}_{\epsilon, \boldsymbol{P}}^{\perp} .
$$


Proof. The proof is similar to Proposition 3.1 in [18] or Proposition 3.1 in [36]. For the sake of completeness, we give a sketch of the proof of injectivity. The surjectivity follows easily from injectivity.

We just need to prove (2.16). Suppose that (2.16) is not true. Then there exists $\epsilon_{k} \rightarrow 0$, $\boldsymbol{P}_{k} \rightarrow \boldsymbol{P} \in \bar{B}_{\delta}\left(\boldsymbol{P}_{0}\right), \Phi_{k} \in \mathcal{K}_{\epsilon, \boldsymbol{P}_{k}}^{\perp}$ such that

$$
\begin{array}{cl}
\tilde{L}_{\epsilon, \boldsymbol{P}_{k}} \Phi_{k}=f_{k}, & \left\|f_{k}\right\|_{t, 0,2+\beta} \rightarrow 0, \\
\Phi_{k} \in \mathcal{K}_{\epsilon, \boldsymbol{P}_{k}}^{\perp}, & \left\|\Phi_{k}\right\|_{t, 2, \beta}=1 .
\end{array}
$$

Let

$$
h_{k}=-p\left(w_{\epsilon, \boldsymbol{P}_{k}}-1\right)_{+}^{p-1} \Phi_{k}, f_{k, 1}=f_{k}+\Pi_{\epsilon, \boldsymbol{P}_{k}} \circ L_{\epsilon, \boldsymbol{P}_{k}}\left(\Phi_{k}\right) .
$$

Then $\Phi_{k}$ satisfies

$$
\begin{cases}\epsilon^{2} \Delta \Phi_{k}=h_{k}+f_{k, 1}, & \text { in } \Omega, \\ \Phi_{k}=0, & \text { on } \partial \Omega .\end{cases}
$$

Note that

$$
\begin{aligned}
\epsilon^{-N} \int_{\Omega} L_{\epsilon_{k}, \boldsymbol{P}_{k}}\left(\Phi_{k}\right) \partial_{i}^{j} w_{\epsilon_{k}, \boldsymbol{P}_{k}} & =\epsilon_{k}^{-N} \int_{\Omega}\left[p\left(w_{\epsilon_{k}, \boldsymbol{P}_{k}}-1\right)_{+}^{p-1} \partial_{i}^{j} w_{\epsilon_{k}, \boldsymbol{P}_{k}}-p\left(w_{j}-1\right)_{+}^{p-1} \partial_{i}^{j} w_{j}\right] \Phi_{k} \\
& =O\left(\epsilon_{k}^{N-2}\right) .
\end{aligned}
$$

Hence

$$
\left\|\Pi_{\epsilon_{k}, \boldsymbol{P}_{k}} \circ L_{\epsilon_{k}, \boldsymbol{P}_{k}}\left(\Phi_{k}\right)\right\|_{t, 0,2+\beta}=O\left(\epsilon_{k}^{N-2}\right)=o(1)
$$

and

$$
\left\|f_{k, 1}\right\|_{t, 0,2+\beta}=o(1)
$$

Let

$$
\Phi_{k}^{j}(y)=\Phi_{k}\left(\epsilon y+P_{k}^{j}\right) \chi\left(x-P_{k}^{j}\right), \quad j=1, \ldots, K,
$$

where $\chi(x)=1$ for $|x| \leqslant \frac{1}{2} \delta$ and $\chi(x)=0$ for $|x| \geqslant \delta$.

Without loss of generality, we may assume that $j=1$. Then $\Phi_{k}^{1}$ satisfies

$$
\begin{cases}\Delta \Phi_{k}^{1}+p\left(w_{\epsilon, P_{k}^{1}}+\sum_{j=2}^{K} w_{\epsilon, P_{k}^{j}}-1\right)_{+}^{p-1} \Phi_{k}^{1}=\tilde{f}_{k}, & \text { in } \Omega_{\epsilon, P^{1}} \\ \Phi_{k}^{1}=0, & \text { on } \partial \Omega_{\epsilon, P^{1}}\end{cases}
$$

where $\tilde{f}_{k}=\chi f_{k, 1}-2 \epsilon^{2} \nabla_{x} \chi \nabla_{x} \Phi_{k}-\epsilon^{2} \Delta \chi \Phi_{k}$. Note that

$$
\left|\epsilon^{2} \nabla_{x} \chi \nabla_{x} \Phi_{k}\right| \leqslant \epsilon\left|\nabla_{y} \Phi_{k}\right|, \quad\left|\epsilon^{2} \Delta \chi \Phi_{k}\right| \leqslant \epsilon^{2}\left|\Phi_{k}\right| .
$$

Hence we have

$$
\left\|\tilde{f}_{k}\right\|_{L_{\beta+2}^{t}\left(\Omega_{\epsilon, P^{1}}\right)} \leqslant C\left\|f_{k, 1}\right\|_{t, 0,2+\beta}+C\left\|\Phi_{k}\right\|_{t, 2, \beta} \leqslant C .
$$

Applying Lemma 2.6, we have

$$
\left\|\Phi_{k}^{1}\right\|_{W_{2, \beta}^{t}\left(\Omega_{\epsilon, P^{1}}\right)} \leqslant C
$$


Let $\Phi_{k}^{1} \rightarrow \Phi_{0}$ weakly in $W_{2, \beta}^{t}\left(R^{N}\right), \Phi_{k}^{1} \stackrel{\text { strong }}{\longrightarrow} \Phi_{0}$ in $L_{\beta}^{t}\left(R^{N}\right)$. Then $\Phi_{0}$ satisfies

$$
\Delta \Phi_{0}+p(w-1)_{+}^{p-1} \Phi_{0}=0, \quad \Phi_{0} \in W_{2, \beta}^{t}\left(R^{N}\right) .
$$

By Lemma 2.4,

$$
\Phi_{0} \in \operatorname{Kernel}(L)=\operatorname{span}\left\{\frac{\partial w}{\partial y_{i}} \mid i=1, \ldots, N\right\}
$$

However,

$$
0=\epsilon^{1-N} \int_{\Omega} \Phi_{k} \frac{\partial w_{\epsilon, P^{1}}}{\partial P_{i}^{1}} \rightarrow-\int_{R^{N}} \Phi_{0} \frac{\partial w}{\partial y_{i}}=0, \quad i=1, \ldots, N .
$$

(Here we have used the fact that $\left(\partial w / \partial y_{i}\right) \in L_{-\beta}^{t^{\prime}}\left(R^{N}\right)$ for $\beta>0>1-(N / t)$.) Hence, $\Phi_{0} \equiv 0$ and $\left\|\Phi_{k}^{1}\right\|_{L_{\beta}^{t}(U)} \rightarrow 0$ for any compact set $U$.

In conclusion, we have obtained that

$$
\left\|\Phi_{k}\right\|_{L_{\beta}^{t}(U)} \rightarrow 0
$$

for any bounded set $U \subset \Omega_{\epsilon, P^{j}}, j=1, \ldots, K$.

Going back to the equation for $\Phi_{k}$, we now have

$$
\begin{aligned}
\left\|h_{k}\right\|_{t, 2+\beta} & \leqslant C \sum_{j=1}^{K}\left\|\left(w_{\epsilon, \boldsymbol{P}_{k}}-1\right)_{+}^{p-1} \Phi_{k}^{j}\right\|_{L_{2+\beta}^{t}\left(\Omega_{\epsilon, P^{j}}\right)} \\
& \leqslant C \sum_{j=1}^{K}\left\|\left(w_{\epsilon, \boldsymbol{P}_{k}}-1\right)_{+}^{p-1} \Phi_{k}^{j}\right\|_{L_{2+\beta}^{t}\left(B_{2 R_{w}(0)}(0)\right)} \rightarrow 0 .
\end{aligned}
$$

By Lemma 2.6,

$$
\left\|\Phi_{k}\right\|_{t, 2, \beta}=o(1),
$$

which is a contradiction to our assumption that $\left\|\Phi_{k}\right\|_{t, 2, \beta}=1$.

\section{Liapunov-Schmidt reduction}

In this section we use the classical Liapunov-Schmidt reduction method to reduce problem (1.9) to a finite-dimensional problem. Similar procedures can be found in $[\mathbf{1 8}],[\mathbf{1 9}]$, $[\mathbf{3 6}]$ and $[34]$, among others.

Recall that $(N /(N-1))<t<N, 0<\beta<\left(N / t^{\prime}\right)-1$. Let

$$
S_{\epsilon}(u):=\epsilon^{2} \Delta u+(u-1)_{+}^{p} .
$$

We first solve the following equation

$$
S_{\epsilon}(u)=0, u \in X_{\epsilon, \boldsymbol{P}}^{t, \beta}
$$

Setting $u=w_{\epsilon, \boldsymbol{P}}+\Phi$ and substituting into $S_{\epsilon}(u)$, we have

$$
S_{\epsilon}(u)=S_{\epsilon}\left(w_{\epsilon, \boldsymbol{P}}\right)+L_{\epsilon, \boldsymbol{P}}(\Phi)+N_{\epsilon, \boldsymbol{P}}(\Phi),
$$


where

$$
\begin{aligned}
L_{\epsilon, \boldsymbol{P}} & =S_{\epsilon}^{\prime}\left(w_{\epsilon, \boldsymbol{P}}\right)=\epsilon^{2} \Delta+p\left(w_{\epsilon, \boldsymbol{P}}-1\right)_{+}^{p-1}, \\
N_{\epsilon, \boldsymbol{P}}(\Phi): & =S\left(w_{\epsilon, \boldsymbol{P}}+\Phi\right)-S\left(w_{\epsilon, \boldsymbol{P}}\right)-L_{\epsilon, \boldsymbol{P}}(\Phi) \\
& =\left(w_{\epsilon, \boldsymbol{P}}+\Phi-1\right)_{+}^{p}-\left(w_{\epsilon, \boldsymbol{P}}-1\right)_{+}^{p}-p\left(w_{\epsilon, \boldsymbol{P}}-1\right)_{+}^{p-1} \Phi .
\end{aligned}
$$

Set

$$
\mathcal{B}_{\eta}=\left\{u \in X_{\epsilon, \boldsymbol{P}}^{t, \beta} \mid\|u\|_{t, 2, \beta}<\eta\right\} .
$$

We then have the following lemma.

Lemma 3.1. For $\epsilon$ and $\eta$ sufficiently small, we have

(1) $\left\|N_{\epsilon, \boldsymbol{P}}(\Phi)\right\|_{t, 0,2+\beta} \leqslant C\|\Phi\|_{t, 2, \beta}^{1+\sigma}, \forall \Phi \in \mathcal{B}_{\eta}$;

(2) $\left\|N_{\epsilon, \boldsymbol{P}}\left(\Phi_{1}\right)-N_{\epsilon, \boldsymbol{P}}\left(\Phi_{2}\right)\right\|_{t, 0,2+\beta} \leqslant C \eta^{\sigma}\left\|\Phi_{1}-\Phi_{2}\right\|_{t, 2, \beta}, \forall \Phi_{1} \in \mathcal{B}_{\eta}, \Phi_{2} \in \mathcal{B}_{\eta}$,

(3) $\left\|S\left(w_{\epsilon, \boldsymbol{P}}\right)\right\|_{t, 0,2+\beta} \leqslant C \epsilon^{N-2}$,

where $\sigma=\min (1, p-1)$.

Proof. Item (3) follows from Lemma 2.3.

For (1), we note that for $t>(N /(N-1)) \geqslant \frac{1}{2} N, \beta>0$, we have, by the Sobolev embedding theorem,

$$
|\Phi|_{L^{\infty}(\Omega)} \leqslant C\|\Phi\|_{t, 2, \beta} \leqslant C \eta .
$$

We can choose $\eta$ so small that

$$
|\Phi|_{L^{\infty}(\Omega)}<\frac{1}{2}
$$

Hence we obtain

$$
\operatorname{support}\left(w_{\epsilon, \boldsymbol{P}}+\Phi-1\right)_{+}^{p} \subset \bigcup_{j=1}^{K} B_{R_{0}^{\prime} \epsilon}\left(P^{j}\right),
$$

where $R_{0}^{\prime}>0$ is a fixed number.

For each $j=1, \ldots, K, x \in B_{R_{0}^{\prime} \epsilon}\left(P^{j}\right)$, we have

$$
\left|N_{\epsilon, \boldsymbol{P}}(\Phi)(x)\right| \leqslant C|\Phi(x)|^{1+\sigma} \leqslant C\|\Phi\|_{t, 2, \beta}^{1+\sigma} .
$$

Because of (3.4), we have

$$
\left\|N_{\epsilon, \boldsymbol{P}}(\Phi)\right\|_{t, 0,2+\beta} \leqslant C\|\Phi\|_{t, 2, \beta}^{1+\sigma} .
$$

We can prove (2) in a similar way.

We are now ready to prove the following main result in this section.

Proposition 3.2. For $\epsilon$ and $\delta$ sufficiently small and $\boldsymbol{P} \in B_{\delta}\left(\boldsymbol{P}_{0}\right)$, the following problem

$$
\Pi_{\epsilon, \boldsymbol{P}}^{\perp} \circ S_{\epsilon}\left(w_{\epsilon, \boldsymbol{P}}+\Phi\right)=0, \quad \Phi \in \mathcal{K}_{\epsilon, \boldsymbol{P}}^{\perp}
$$

has a unique solution:

$$
\Phi:=\Phi_{\epsilon, \boldsymbol{P}} \in \mathcal{B}_{\epsilon^{(1-\delta)(N-2)}}
$$


Proof. We write (3.5) in the following form:

$$
\begin{aligned}
0 & =\Pi_{\epsilon, \boldsymbol{P}}^{\perp} \circ\left(S_{\epsilon}\left(w_{\epsilon, \boldsymbol{P}}\right)+L_{\epsilon, \boldsymbol{P}}(\Phi)+N_{\epsilon, \boldsymbol{P}}(\Phi)\right) \\
& =\Pi_{\epsilon, \boldsymbol{P}}^{\perp} \circ L_{\epsilon, \boldsymbol{P}}(\Phi)+\Pi_{\epsilon, \boldsymbol{P}}^{\perp} \circ S_{\epsilon}\left(w_{\epsilon, \boldsymbol{P}}\right)+\Pi_{\epsilon, \boldsymbol{P}}^{\perp} \circ N_{\epsilon, \boldsymbol{P}}(\Phi) .
\end{aligned}
$$

Therefore, (3.5) is equivalent to

$$
\Phi=G_{\epsilon}(\Phi):=-\tilde{L}_{\epsilon, \boldsymbol{P}}^{-1} \circ\left(\Pi_{\epsilon, \boldsymbol{P}}^{\perp} \circ S_{\epsilon}\left(w_{\epsilon, \boldsymbol{P}}\right)+\Pi_{\epsilon, \boldsymbol{P}}^{\perp} \circ N_{\epsilon, \boldsymbol{P}}(\Phi)\right) .
$$

By Proposition 2.8 and parts (1) and (3) of Lemma 3.1, we have, for $\Phi \in \mathcal{B}_{\eta}$, where $\eta=\epsilon^{(1-\delta)(N-2)}$,

$$
\begin{aligned}
\left\|G_{\epsilon}(\Phi)\right\|_{t, 2, \beta} & \leqslant C\left\|S_{\epsilon}\left(w_{\epsilon, \boldsymbol{P}}\right)\right\|_{t, 0,2+\beta}+C\left\|N_{\epsilon}(\Phi)\right\|_{t, 0,2+\beta} \\
& \leqslant C \epsilon^{N-2}+C \epsilon^{(1+\sigma)(1-\delta)(N-2)}<\frac{1}{2} \eta .
\end{aligned}
$$

Moreover, by Lemma $3.1(2)$,

$$
\left\|G_{\epsilon}\left(\Phi_{1}\right)-G_{\epsilon}\left(\Phi_{1}\right)\right\|_{t, 2, \beta} \leqslant C \eta^{\sigma}\left\|\Phi_{1}-\Phi_{2}\right\|_{t, 2, \beta}<\frac{1}{2}\left\|\Phi_{1}-\Phi_{2}\right\|_{t, 2, \beta} .
$$

Thus $G_{\epsilon}$ is a contraction map from $\mathcal{B}_{\eta}$ to $\mathcal{B}_{\eta}$.

By the contraction mapping principle (see [18], Proposition 6.5 in [34] and Lemma 3.3 in $[\mathbf{3 6}]$ for a similar approach), Proposition 3.2 can be easily proved. We omit the technical details.

We next expand $\Phi_{\epsilon, \boldsymbol{P}}$ in terms of $\epsilon^{N-2}$. To this end, we set $\Phi_{\epsilon, \boldsymbol{P}}^{0}$ to be defined as follows

$$
\Phi_{\epsilon, \boldsymbol{P}}^{0}(x)=k_{w} \sum_{j=1}^{K} F_{j}\left(\boldsymbol{P}, P^{j}\right) \Phi_{0}\left(\frac{x-P^{j}}{\epsilon}\right),
$$

where $\Phi_{0}$ is the unique solution of the following problem

$$
\begin{cases}\Delta \phi_{0}+p(w-1)_{+}^{p-1} \Phi_{0}+p(w-1)_{+}^{p-1}=0, & \text { in } R^{N}, \\ \Phi_{0}(y)=\Phi_{0}(|y|), \quad \Phi_{0}(|y|) \rightarrow 0, & \text { as }|y| \rightarrow+\infty .\end{cases}
$$

It is easy to see that $\Phi_{0}(|y|)=C_{0}|y|^{2-N}$ for $|y| \geqslant R_{w}$, where $C_{0}$ is a constant. Remarkably, we can write $\Phi_{0}$ explicitly.

Lemma 3.3. Let $\Phi_{0}$ be the unique solution of (3.7). We then have

$$
\Phi_{0}=-w-\frac{1}{2}(p-1) r w^{\prime}(r) .
$$

Thus

$$
C_{0}=\frac{k_{w}}{(N-2)\left|S^{N-1}\right|}\left(\frac{1}{2}(p-1)(N-2)-1\right) .
$$

Proof. By invariance of the equation $\Delta u+u^{p}=0$, it is easy to see that $-((w-1)+$ $\left.\frac{1}{2}(p-1) r(w-1)^{\prime}\right)$ satisfies $\Delta u+p(w-1)_{+}^{p-1} u=0$ in $B_{R_{w}}(0)$. Hence $-w-\frac{1}{2}(p-1) r w^{\prime}(r)$ satisfies equation (3.7). By uniqueness, $\Phi_{0}=-w-\frac{1}{2}(p-1) r w^{\prime}(r)$. The formula for $C_{0}$ follows from Lemma 2.1 . 
Let

$$
\tilde{\Phi}_{\epsilon, \boldsymbol{P}}^{0}(x):=k_{w} \sum_{j=1}^{K} F_{j}\left(\boldsymbol{P}, P^{j}\right)\left(\Phi_{0}\left(\frac{x-P^{j}}{\epsilon}\right)-C_{0}(N-2)\left|S^{N-1}\right| \epsilon^{N-2} H\left(x, P^{j}\right)\right),
$$

be the projection of $\Phi_{\epsilon, \boldsymbol{P}}(x)$ into $H_{0}^{1}(\Omega)$. Note that

$$
\Phi_{\epsilon, \boldsymbol{P}}^{0}(x)=\tilde{\Phi}_{\epsilon, \boldsymbol{P}}^{0}(x)+O\left(\epsilon^{N-2}\right) .
$$

We have the following lemma.

Lemma 3.4. Let $\Phi_{\epsilon, \boldsymbol{P}}$ be defined by Proposition 3.2. Then

$$
\left\|\Phi_{\epsilon, \boldsymbol{P}}-\epsilon^{N-2} \tilde{\Phi}_{\epsilon, \boldsymbol{P}}^{0}\right\|_{t, 2, \beta}=O\left(\epsilon^{N-2+\delta_{0}}\right),
$$

where $\tilde{\Phi}_{\epsilon, \boldsymbol{P}}^{0}$ is defined by (3.8) and $\delta_{0}>0$ is a positive constant.

Proof. We first note that, by (2.6),

$$
S_{\epsilon}\left(w_{\epsilon, \boldsymbol{P}}\right)=p k_{w}(w(y)-1)_{+}^{p-1} F_{j}\left(\boldsymbol{P}, P^{j}\right) \epsilon^{N-2}+O\left(\epsilon^{N-1}\right)
$$

for $x \in B_{R_{0} \epsilon}\left(P^{j}\right)$. Thus

$$
\Pi_{\epsilon, \boldsymbol{P}}^{\perp} \circ S_{\epsilon}\left(w_{\epsilon, \boldsymbol{P}}(x)\right)=p k_{w}(w-1)_{+}^{p-1} F_{j}\left(\boldsymbol{P}, P^{j}\right) \epsilon^{N-2}+O\left(\epsilon^{N-1}\right)
$$

for $x \in B_{R_{0} \epsilon}\left(P^{j}\right)$. Note also that

$$
\left\|\Pi_{\epsilon, \boldsymbol{P}}^{\perp} \circ N_{\epsilon}\left(\Phi_{\epsilon, \boldsymbol{P}}\right)\right\|_{t, 0,2+\beta}=O\left(\epsilon^{(1+\sigma)(1-\delta)(N-2)}\right)=O\left(\epsilon^{N-2+\delta_{0}}\right) .
$$

On the other hand, $\Phi_{\epsilon, \boldsymbol{P}}^{0} \perp X_{0},(3.9)$ can be proved by using equation (3.5). Please see the proof of Lemma A 1 in Appendix A for similar proofs.

\section{The reduced problem: proof of Theorem 1.4}

By Proposition 3.2, there exists a unique $\Phi_{\epsilon, \boldsymbol{P}}$ such that

$$
S_{\epsilon}\left(w_{\epsilon, \boldsymbol{P}}+\Phi_{\epsilon, \boldsymbol{P}}\right) \in C_{\epsilon, \boldsymbol{P}}, \Phi_{\epsilon, \boldsymbol{P}} \in \mathcal{K}_{\epsilon, \boldsymbol{P}}^{\perp}
$$

In other words, we have

$$
S_{\epsilon}\left(w_{\epsilon, \boldsymbol{P}}+\Phi_{\epsilon, \boldsymbol{P}}\right)=\sum_{k, l} \beta_{k}^{l}(\boldsymbol{P}) \partial_{k}^{l} w_{\epsilon, \boldsymbol{P}}
$$

for some constants $\beta_{k}^{l}(\boldsymbol{P}), k=1, \ldots, N, l=1, \ldots, K$. (Here the summation is for $k=1, \ldots, N, l=1, \ldots, K$.)

Set

$$
W_{\epsilon, k}^{l}(\boldsymbol{P}):=\epsilon^{-N} \int_{\Omega} S_{\epsilon}\left(w_{\epsilon, \boldsymbol{P}}+\Phi_{\epsilon, \boldsymbol{P}}\right) \partial_{k}^{l} w_{\epsilon, \boldsymbol{P}}, \quad k=1, \ldots, N, \quad l=1, \ldots, K .
$$

Therefore, to solve (3.2), it is enough to find out a zero of $W_{\epsilon, k}^{l}(\boldsymbol{P})$. We need to compute $W_{\epsilon, k}^{l}(\boldsymbol{P})$. We have the following lemma. 
Lemma 4.1. For $\epsilon \ll 1$, it holds that

$$
W_{\epsilon, i}^{j}(\boldsymbol{P})=c_{w} \epsilon^{N-2} \partial_{i}^{j} F(\boldsymbol{P})+O\left(\epsilon^{N-2+\delta_{0}}\right), \quad i=1, \ldots, N, \quad j=1, \ldots, K,
$$

where $c_{w} \neq 0$ is a generic constant given by

$$
c_{w}=-k_{w} \frac{1}{2} \int_{R^{N}}(w-1)_{+}^{p} .
$$

Proof of Lemma 4.1. Note that for $i=1, \ldots, N, j=1, \ldots, K$,

$$
\begin{aligned}
\partial_{i}^{j} w_{\epsilon, \boldsymbol{P}}=\partial_{i}^{j} w_{\epsilon, P^{j}}, & W^{j}=\epsilon_{\Omega} S_{\epsilon}\left(w_{\epsilon, \boldsymbol{P}}+\Phi_{\epsilon, \boldsymbol{P}}\right) \partial_{i}^{j} w_{\epsilon, P^{j}} \\
& =\int_{\Omega_{\epsilon, P^{j}}} S_{\epsilon}\left(w_{\epsilon, \boldsymbol{P}}\right) \partial_{i}^{j} w_{\epsilon, P^{j}}+\int_{\Omega_{\epsilon, P^{j}}} L_{\epsilon, \boldsymbol{P}}\left(\Phi_{\epsilon, \boldsymbol{P}}\right) \partial_{i}^{j} w_{\epsilon, P^{j}}+\int_{\Omega_{\epsilon, P^{j}}} N_{\epsilon}\left(\Phi_{\epsilon, \boldsymbol{P}}\right) \partial_{i}^{j} w_{\epsilon, P^{j}} \\
& =J_{1}+J_{2}+J_{3},
\end{aligned}
$$

where the $J_{i}, i=1,2,3$, are defined at the last equality.

We first estimate $J_{2}$ and $J_{3}$. Note that

$$
\begin{aligned}
J_{2} & =\int_{\Omega_{\epsilon, P} j} p\left[\left(w_{\epsilon, \boldsymbol{P}}+\Phi_{\epsilon, \boldsymbol{P}}-1\right)_{+}^{p-1} \partial_{i}^{j} w_{\epsilon, P^{j}}-\left(w_{j}-1\right)_{+}^{p-1} \partial_{i}^{j} w_{j}\right] \Phi_{\epsilon, \boldsymbol{P}} \\
& =O\left(\epsilon^{N-2+\delta_{0}}\right)
\end{aligned}
$$

by Lemmas 2.3 and 3.4, and

$$
J_{3}=\int_{\Omega_{\epsilon, P^{j}}} N_{\epsilon}\left(\Phi_{\epsilon, \boldsymbol{P}}\right) \partial_{i}^{j} w_{\epsilon, P^{j}}=O\left(\epsilon^{N-2+\delta_{0}}\right) .
$$

It remains to estimate $J_{1}$. By Lemma 3.4 and the fact that

$$
\partial_{i}^{j} w_{\epsilon, P^{j}}=\partial_{i}^{j} w\left(\frac{x-P^{j}}{\epsilon}\right)-\epsilon^{N-2} k_{w} \partial_{i}^{j} H\left(x, P^{j}\right),
$$

we have

$$
\begin{aligned}
J_{1} & =\int_{\Omega_{\epsilon, P^{j}}} S_{\epsilon}\left(w_{\epsilon, \boldsymbol{P}}\right) \partial_{i}^{j} w_{\epsilon, P^{j}} \\
& =\int_{\Omega_{\epsilon, P^{j}}} S_{\epsilon}\left(w_{\epsilon, \boldsymbol{P}}\right) \partial_{i}^{j} w_{j}+O\left(\epsilon^{2(N-2)}\right) \\
& =\left.k_{w} \sum_{k=1}^{N} \frac{\partial}{\partial x_{k}} F_{j}(\boldsymbol{P}, x)\right|_{x=P^{j}} \epsilon^{N-2} \int_{R^{N}} \frac{\partial w}{\partial y_{i}} y_{k} p(w-1)_{+}^{p-1} \mathrm{~d} y+O\left(\epsilon^{N-2+\delta_{0}}\right) \\
& =\left.k_{w} \frac{\partial}{\partial x_{i}} F_{j}(\boldsymbol{P}, x)\right|_{x=P^{j}} \epsilon^{N-2} \int_{R^{N}} \frac{\partial w}{\partial y_{i}} y_{i} p(w-1)_{+}^{p-1} \mathrm{~d} y+O\left(\epsilon^{N-2+\delta_{0}}\right) \\
& =c_{w} \epsilon^{N-2} \partial_{i}^{j} F(\boldsymbol{P})+O\left(\epsilon^{N-2+\delta_{0}}\right)
\end{aligned}
$$


since

$$
\int_{R^{N}} \frac{\partial w}{\partial y_{i}} y_{i} p(w-1)_{+}^{p-1}=\int_{R^{N}} \frac{\partial(w-1)_{+}^{p}}{\partial y_{i}} y_{i}=-\int_{R^{N}}(w-1)_{+}^{p},
$$

and

$$
\left.\frac{\partial}{\partial x_{i}} F_{j}(\boldsymbol{P}, x)\right|_{x=P^{j}}=\frac{1}{2} \partial_{i}^{j} F(\boldsymbol{P}) .
$$

Finally we prove Theorem 1.4.

Proof of Theorem 1.4. By Proposition 3.2 we have

$$
S_{\epsilon}\left(w_{\epsilon, \boldsymbol{P}}+\Phi_{\epsilon, \boldsymbol{P}}\right)=\sum_{k, l} \beta_{k}^{l}(\boldsymbol{P}) \partial_{k}^{l} w_{\epsilon, \boldsymbol{P}}
$$

By Lemma 4.1 we have

$$
\beta_{k}^{l}(\boldsymbol{P})=(1+o(1)) \epsilon^{2} W_{\epsilon, k}^{l}(\boldsymbol{P})=\epsilon^{N}\left(c_{w} \partial_{k}^{l} F(\boldsymbol{P})+o(1)\right) .
$$

Since $\boldsymbol{P}_{0}$ is a non-degenerate critical point of $F(\boldsymbol{P})$, by Brouwer's fixed point theorem, there exists a $\boldsymbol{P}_{\epsilon}$ such that

$$
\beta_{k}^{l}\left(\boldsymbol{P}_{\epsilon}\right)=0, \quad k=1, \ldots, N, \quad l=1, \ldots, K .
$$

In other words, we have that $u_{\epsilon}=w_{\epsilon, \boldsymbol{P}}+\Phi_{\epsilon, \boldsymbol{P}} \in X^{t, \beta}$ satisfies

$$
\epsilon^{2} \Delta u_{\epsilon}+\left(u_{\epsilon}-1\right)_{+}^{p}=0, \quad \text { in } \Omega,
$$

and $u_{\epsilon}=0$ on $\partial \Omega$. By the standard regularity theorem, we have $u_{\epsilon} \in C^{2}(\Omega) \cap C^{0}(\bar{\Omega})$.

It remains to check that $u_{\epsilon}>0$ in $\Omega$. By the Maximum Principle, it is enough to show that $u_{\epsilon}^{-} \equiv 0$. Multiplying the equation by $u_{\epsilon}^{-}$and integrating by parts we have

$$
\epsilon^{2} \int_{\Omega}\left|\nabla u_{\epsilon}^{-}\right|^{2}=\int_{\Omega} u_{\epsilon}^{-}\left(u_{\epsilon}-1\right)_{+}^{p}=0,
$$

which implies that $u_{\epsilon}^{-} \equiv 0$.

By following the same arguments as in Steps 13 and 14 in [20], we have that $u_{\epsilon}$ has exactly $K$ local maximum points $P_{\epsilon}^{j}$ and, moreover, $P_{\epsilon}^{j} \rightarrow P_{0}^{j}, j=1, \ldots, K$. Furthermore, the set $A_{\epsilon}=\left\{u_{\epsilon}>1\right\}$ consists of exactly $K$ components and (1.11) holds for $R_{1}>R_{2}$, where $R_{1}=R_{w}+o(1), R_{2}=R_{w}+o(1)$.

\section{Uniqueness of $u_{\epsilon}$}

Let $u_{\epsilon}$ be the solution constructed in $\S 4$. In this section, we prove the uniqueness of $u_{\epsilon}$.

Let $w_{\epsilon, \boldsymbol{P}}, \mathcal{K}_{\epsilon, \boldsymbol{P}}, \mathcal{C}_{\epsilon, \boldsymbol{P}}, \mathcal{K}_{\epsilon, \boldsymbol{P}}^{\perp}, \mathcal{C}_{\epsilon, \boldsymbol{P}}^{\perp}$ and $S_{\epsilon}(u)$ be as defined in $\S 2$.

For any $u, v \in H_{0}^{1}(\Omega)$, we define

$$
\langle u, v\rangle_{\epsilon}=\epsilon^{-N} \int_{\Omega}\left(\epsilon^{2} \nabla u \cdot \nabla v\right), \quad\|u\|_{\epsilon}=\langle u, v\rangle_{\epsilon}^{1 / 2} .
$$


For any $u \in H_{0}^{1}(\Omega)$, we define the energy functional associated with (1.8) as

$$
J_{\epsilon}(u)=\epsilon^{-N}\left[\frac{1}{2} \epsilon^{2} \int_{\Omega}|\nabla u|^{2}-\int_{\Omega} F(u)\right],
$$

where $F(u)=\int_{0}^{u} f(s) \mathrm{d} s$.

Fix any $\boldsymbol{P} \in \Omega^{K}$. We define

$$
K_{\epsilon}(\boldsymbol{P})=J_{\epsilon}\left(w_{\epsilon, \boldsymbol{P}}+\Phi_{\epsilon, \boldsymbol{P}}\right),
$$

where $\Phi_{\epsilon, \boldsymbol{P}}$ is the unique solution given by Proposition 3.2.

Let $\boldsymbol{P}_{0}$ be a non-degenerate critical point of $F(\boldsymbol{P})$. Set

$$
\Lambda=B_{\delta}(\boldsymbol{P}) \subset \Omega^{K} .
$$

Let $K_{\epsilon}(\boldsymbol{P})$ be defined by (5.2). Then we have the following lemma.

Lemma 5.1. $u_{\epsilon}=w_{\epsilon, \boldsymbol{P}}+\Phi_{\epsilon, \boldsymbol{P}}$ is a solution of (1.9) if and only if $\boldsymbol{P}$ is a critical point of $K_{\epsilon}(\boldsymbol{P})$ in $\Lambda$.

Proof. The proof is similar to Proposition 3.5 in [18].

Let $u_{\epsilon}$ be a single-condensation solution with the unique local maximum $\boldsymbol{P}_{\epsilon} \rightarrow \boldsymbol{P}_{0}$. By Lemma 5.1, we have $u_{\epsilon}=w_{\epsilon, \boldsymbol{Q}_{\epsilon}}+\Phi_{\epsilon, \boldsymbol{Q}_{\epsilon}}$ for some $\boldsymbol{Q}_{\epsilon} \in \Lambda, \Phi_{\epsilon, \boldsymbol{Q}_{\epsilon}} \in \mathcal{K}_{\epsilon, \boldsymbol{Q}_{\epsilon}}^{\perp}$, and $\boldsymbol{Q}_{\epsilon}$ is a critical point of $K_{\epsilon}(\boldsymbol{P})$ in $\Lambda$.

By Lemma 5.1, to prove the uniqueness of $u_{\epsilon}$, we just need to show that $K_{\epsilon}(\boldsymbol{P})$ has only one critical point in $\Lambda$.

Let us define

$$
\tilde{K}_{\epsilon}(\boldsymbol{P})=K\left(\frac{1}{2} \int_{R^{N}}|\nabla w|^{2}-\int_{R^{N}} F(w)\right)-c_{w} F(\boldsymbol{P}) \epsilon^{N-2} .
$$

It is easy to see that $\tilde{K}_{\epsilon}(\boldsymbol{P})$ has only one critical point, $\boldsymbol{P}_{0}$, in $\Lambda$ if $\delta$ is small.

We now compute $\nabla K_{\epsilon}(\boldsymbol{P})$. First we have the following lemma.

Lemma 5.2. For $\boldsymbol{P} \in \Lambda$, we have

$$
\nabla K_{\epsilon}(\boldsymbol{P})=\nabla \tilde{K}_{\epsilon}(\boldsymbol{P})+o\left(\epsilon^{N-2}\right) .
$$

Proof. Observe that

$$
\begin{aligned}
\partial_{i}^{j} K_{\epsilon}(\boldsymbol{P}) & =\left\langle w_{\epsilon, \boldsymbol{P}}+\Phi_{\epsilon, \boldsymbol{P}}, \partial_{i}^{j}\left(w_{\epsilon, \boldsymbol{P}}+\Phi_{\epsilon, \boldsymbol{P}}\right)\right\rangle_{\epsilon}-\epsilon^{-N} \int_{\Omega}\left(w_{\epsilon, \boldsymbol{P}}+\Phi_{\epsilon, \boldsymbol{P}}\right)^{p} \partial_{i}^{j}\left(w_{\epsilon, \boldsymbol{P}}+\Phi_{\epsilon, \boldsymbol{P}}\right) \\
& =\epsilon^{-N} \int_{\Omega} S_{\epsilon}\left(w_{\epsilon, \boldsymbol{P}}+\Phi_{\epsilon, \boldsymbol{P}}\right) \partial_{i}^{j}\left(w_{\epsilon, \boldsymbol{P}}+\Phi_{\epsilon, \boldsymbol{P}}\right) \\
& =\sum_{k, l} \beta_{k}^{l}(\boldsymbol{P}) \int_{\Omega_{\epsilon}} \partial_{k}^{l} w_{\epsilon, \boldsymbol{P}} \partial_{i}^{j}\left(w_{\epsilon, \boldsymbol{P}}+\Phi_{\epsilon, \boldsymbol{P}}\right) \\
= & \partial_{i}^{j} \tilde{K}_{\epsilon}(\boldsymbol{P})+o\left(\epsilon^{N-2}\right),
\end{aligned}
$$

by Lemma 4.1. 
The following lemma shows that a critical point of $K_{\epsilon}$ is non-degenerate.

Lemma 5.3. Let $\boldsymbol{Q}_{\epsilon}$ be a critical point of $K_{\epsilon}(\boldsymbol{P})$ over $\Lambda$. Then we have

$$
\left.\partial_{i}^{j} \partial_{k}^{l} K_{\epsilon}(\boldsymbol{P})\right|_{\boldsymbol{P}=\boldsymbol{Q}_{\epsilon}}=\left.\partial_{i}^{j} \partial_{k}^{l} \tilde{K}_{\epsilon}(\boldsymbol{P})\right|_{\boldsymbol{P}=\boldsymbol{Q}_{\epsilon}}+o\left(\epsilon^{N-2}\right) .
$$

The proof of Lemma 5.3 is very complicated and is thus left to Appendix A. Let us now use it to prove the uniqueness of $u_{\epsilon}$.

The proof of the uniqueness of $u_{\epsilon}$ is completed by the following lemma.

Lemma 5.4. There exists a unique critical point of $K_{\epsilon}(\boldsymbol{P})$ over $\Lambda$.

Proof. As we already know by Theorem 1.4 and Lemma 5.1, $K_{\epsilon}(\boldsymbol{P})$ has a critical point $\boldsymbol{Q}_{\epsilon}$ and any other critical point of $K_{\epsilon}(\boldsymbol{P})$ is in $\Lambda$.

We now show that $Q_{\epsilon}$ is unique.

First, by Lemma 5.3, there are only a finite number of critical points of $K_{\epsilon}(\boldsymbol{P})$ in $\Lambda$. Let $\boldsymbol{Q}_{\epsilon}^{i} \in \Lambda, i=1, \ldots, k_{\epsilon}$, be the critical points, where $k_{\epsilon}$ is the number of critical points. At each critical point $\boldsymbol{Q}_{\epsilon}^{i}$, we have, by Lemmas 5.3 and 5.2,

$$
\operatorname{deg}\left(\nabla K_{\epsilon}, B_{\delta_{i}}\left(\boldsymbol{Q}_{\epsilon}^{i}\right), 0\right)=(-1)^{d_{i}},
$$

where $\delta_{i}>0$ are small constants so that $B_{\delta_{i}}\left(\boldsymbol{Q}_{\epsilon}^{i}\right)(\subset \Lambda)$ contains only one critical point (i.e. $\boldsymbol{Q}_{\epsilon}^{i}$ ) of $\nabla K_{\epsilon}(\boldsymbol{P})$. Here $d_{i}$ is the Morse index of $F(\boldsymbol{P})$ at $\boldsymbol{Q}_{\epsilon}^{i}$. By Lemma $5.3, d_{i}=d$, where $d$ is the Morse index of $F(\boldsymbol{P})$ at $\boldsymbol{P}_{0}$.

Hence by the additivity of the degree we have

$$
\operatorname{deg}\left(\nabla K_{\epsilon}, \Lambda, 0\right)=\sum_{j=1}^{k_{\epsilon}}(-1)^{d_{j}}=k_{\epsilon}(-1)^{d} .
$$

On the other hand, since $\tilde{K}_{\epsilon}(\boldsymbol{P})$ has only one critical point in $\Lambda$ and by Lemma 5.2 , $\nabla K_{\epsilon}(\boldsymbol{P})=\nabla \tilde{K}_{\epsilon}(\boldsymbol{P})+o\left(\epsilon^{N-2}\right)$, by a continuity argument (note that $\nabla K_{\epsilon}(\boldsymbol{P}) \neq 0$ and $\nabla \tilde{K}_{\epsilon}(\boldsymbol{P}) \neq 0$ on $\left.\partial \Lambda\right)$, we obtain

$$
\operatorname{deg}\left(\nabla K_{\epsilon}, \Lambda, 0\right)=\operatorname{deg}\left(\nabla \tilde{K}_{\epsilon}, \Lambda, 0\right)=(-1)^{d} .
$$

Comparing (5.6) and (5.7), we obtain $k_{\epsilon}=1$.

\section{Proofs of Theorems 1.1 and 1.3}

In this section, we prove Theorems 1.1 and 1.3. By Theorem 1.4, for each $\epsilon<\epsilon_{0}$ sufficiently small, there exists a unique solution $u_{\epsilon}$ with $K$ local maximum points $P_{\epsilon}^{j}$, $j=1, \ldots, K$, and $P_{\epsilon}^{j} \rightarrow P_{0}^{j}$ as $\epsilon \rightarrow 0$. To prove Theorems 1.1 and 1.3, it is enough to study the following equation:

$$
I(\epsilon):=\epsilon^{-2 p /(p-1)} \int_{\Omega}\left(u_{\epsilon}-1\right)_{+}^{p}=M .
$$

First we have the following lemma. 
Lemma 6.1. $I(\epsilon)$ is a continuous function for $\epsilon \in\left(0, \epsilon_{0}\right)$.

Proof. This follows from the uniqueness of $u_{\epsilon}$. In fact let $\epsilon \in\left(0, \epsilon_{0}\right)$ and $\epsilon_{k} \rightarrow \epsilon$ as $k \rightarrow$ $+\infty$. Since $u_{\epsilon_{k}}$ is uniformly bounded for $\epsilon_{k} \in\left(0, \epsilon_{0}\right)$, we can assume that $u_{\epsilon_{k}} \rightarrow \tilde{u}_{\epsilon}$, where $\tilde{u}_{\epsilon}$ satisfies (1.9). Moreover, the set $A_{\epsilon_{k}}=\left\{u_{\epsilon_{k}}>1\right\}$ approaches the set $A_{\epsilon}=\left\{\tilde{u}_{\epsilon}>1\right\}$. Therefore, we see that $u_{\epsilon}$ has exactly $K$ local maximum points $P_{j}^{\epsilon}$ with $P_{j}^{\epsilon}=P_{j}^{0}+o(1)$. (We remark here that any local maximum point of $u_{\epsilon}$ is non-degenerate (see [20]).) By uniqueness of $u_{\epsilon}$, we have $\tilde{u}_{\epsilon}=u_{\epsilon}$ and thus $\lim _{\epsilon_{k} \rightarrow \epsilon} I\left(\epsilon_{k}\right)=I(\epsilon)$.

We now compute $I(\epsilon)$.

Lemma 6.2. For $\epsilon$ sufficiently small, we have

$$
\int_{\Omega}\left(u_{\epsilon}-1\right)_{+}^{p}=\epsilon^{N}\left[K \int_{R^{N}}(w-1)_{+}^{p}+\epsilon^{N-2} d_{w} F\left(\boldsymbol{P}^{0}\right)+o\left(\epsilon^{N-2}\right)\right],
$$

where $d_{w}=\left(\frac{1}{2}(p-1)(N-2)-1\right) k_{w}$ is a generic constant.

Remark 6.3. Combining Lemma 6.1 and Lemma 6.4, Theorem 1.4 is proved by taking $I_{p}:=K \int_{R^{N}}(w-1)_{+}^{p}$.

Remark 6.4. Note that when $p=p^{*}, d_{w}=0$.

Proof. Note that

$$
u_{\epsilon}=w_{\epsilon, \boldsymbol{Q}_{\epsilon}}+\Phi_{\epsilon, \boldsymbol{Q}_{\epsilon}}
$$

and

$$
\int_{\Omega}\left(u_{\epsilon}-1\right)_{+}^{p}=\sum_{j=1}^{K} \int_{B_{2 R_{0} \epsilon}\left(Q_{\epsilon}^{j}\right)}\left(u_{\epsilon}-1\right)_{+}^{p}
$$

for some $R_{0}>0$. We just need to compute

$$
\int_{B_{2 R_{0} \epsilon}\left(Q_{\epsilon}^{j}\right)}\left(u_{\epsilon}-1\right)_{+}^{p}, \quad j=1, \ldots, K .
$$

We now compute the case when $j=1$. The others are similar. We obtain

$$
\begin{aligned}
\int_{B_{2 R_{0} \epsilon}\left(Q_{\epsilon}^{1}\right)}\left(u_{\epsilon}-1\right)_{+}^{p} & =\epsilon^{N} \int_{B_{2 R_{0}}(0)}\left(u_{\epsilon}\left(Q_{\epsilon}^{1}+\epsilon y\right)-1\right)_{+}^{p} \\
= & \epsilon^{N} \int_{B_{2 R_{w}}(0)}\left(w_{\epsilon, \boldsymbol{Q}_{\epsilon}}+\Phi_{\epsilon, \boldsymbol{Q}_{\epsilon}}-1\right)_{+}^{p} \\
= & \epsilon^{N}\left[\int_{B_{2 R_{0}}(0)}\left(w(y)+k_{w} \epsilon^{N-2} F_{1}\left(\boldsymbol{Q}_{\epsilon}, Q_{\epsilon}^{1}\right)+\Phi_{\epsilon, \boldsymbol{Q}_{\epsilon}}-1\right)_{+}^{p}+O\left(\epsilon^{N-1}\right)\right] \\
= & \epsilon^{N}\left[\int_{R^{N}}(w-1)_{+}^{p}+p k_{w} \int_{R^{N}}(w-1)_{+}^{p-1} \epsilon^{N-2} F_{1}\left(\boldsymbol{Q}_{\epsilon}, Q_{\epsilon}^{1}\right)\right. \\
& \left.+p \int_{R^{N}}(w-1)_{+}^{p-1} \Phi_{\epsilon, \boldsymbol{Q}_{\epsilon}}+O\left(\epsilon^{N-1}\right)\right] .
\end{aligned}
$$


By Lemma 3.4, for $y \in B_{2 R_{0}}(0)$, we have

$$
\Phi_{\epsilon, \boldsymbol{Q}_{\epsilon}}=k_{w} \epsilon^{N-2} F_{1}\left(\boldsymbol{Q}_{\epsilon}, Q_{\epsilon}^{1}\right) \Phi_{0}\left(\frac{x-Q_{\epsilon}^{1}}{\epsilon}\right)+o\left(\epsilon^{N-2}\right),
$$

where $\Phi_{0}$ is the solution of (3.7).

Thus

$$
p \int_{R^{N}}(w-1)_{+}^{p-1} \Phi_{\epsilon, \boldsymbol{Q}_{\epsilon}}=p \epsilon^{N-2} \int_{R^{N}}(w-1)_{+}^{p-1} F_{1}\left(\boldsymbol{Q}_{\epsilon}, Q_{\epsilon}^{1}\right) k_{w} \Phi_{0}(y)+o\left(\epsilon^{N-2}\right) .
$$

Substituting (6.6) into (6.4) and noting that $\boldsymbol{Q}_{\epsilon} \rightarrow \boldsymbol{P}_{0}$, we obtain

$$
\begin{aligned}
& \int_{B_{2 R_{0} \epsilon}\left(Q_{\epsilon}^{1}\right)}\left(u_{\epsilon}-1\right)_{+}^{p} \\
&=\epsilon^{N}\left[\int_{R^{N}}(w-1)_{+}^{p}+p \int_{R^{N}}(w-1)_{+}^{p-1} k_{w} F_{1}\left(\boldsymbol{P}_{0}, P_{0}^{1}\right) \epsilon^{N-2}\right. \\
&\left.\quad+p \int_{R^{N-1}}(w-1)_{+}^{p-1} \Phi_{0} k_{w} F_{1}\left(\boldsymbol{P}_{0}, P_{0}^{1}\right) \epsilon^{N-2}+o\left(\epsilon^{N-2}\right)\right] \\
& \quad \epsilon^{N}\left[\int_{R^{N}}(w-1)_{+}^{p}+p \int_{R^{N}}(w-1)_{+}^{p-1}\left(\Phi_{0}+1\right) k_{w} F_{1}\left(\boldsymbol{P}_{0}, P_{0}^{1}\right) \epsilon^{N-2}+o\left(\epsilon^{N-2}\right)\right] .
\end{aligned}
$$

From the equation for $\Phi_{0}$,

$$
\begin{aligned}
p \int_{R^{N}}(w-1)_{+}^{p-1}\left(\Phi_{0}+1\right) & =-\int_{\partial B_{R_{w}}(0)} \frac{\partial \Phi_{0}}{\partial \nu} \\
& =-C_{0}(2-N)\left|\partial B_{1}(0)\right|=d_{w}
\end{aligned}
$$

where $\Phi_{0}(r)=C_{0} / r^{N-2}$ for $r \geqslant R_{w}$. By Lemma 3.3,

$$
C_{0}=\frac{k_{w}}{(N-2)\left|S^{N-1}\right|}\left(\frac{1}{2}(p-1)(N-2)-1\right) .
$$

Hence

$$
\int_{B_{2 R_{0} \epsilon}\left(Q_{\epsilon}^{1}\right)}\left(u_{\epsilon}-1\right)_{+}^{p}=\epsilon^{N}\left[\int_{R^{N}}(w-1)_{+}^{p}+d_{w} F_{1}\left(\boldsymbol{P}_{0}, P_{0}^{1}\right) \epsilon^{N-2}+o\left(\epsilon^{N-2}\right)\right] .
$$

Combining the estimates of

$$
\int_{B_{2 R_{0} \epsilon}\left(Q_{\epsilon}^{j}\right)}\left(u_{\epsilon}-1\right)_{+}^{p}, \quad j=1, \ldots, K
$$

equation (6.2) is thus proved.

Finally we can prove Theorems 1.1 and 1.3 . 
Proof of Theorems 1.1 and 1.3. Consider the equation

$$
I(\epsilon)=M
$$

By Lemma 6.1,

$$
I(\epsilon)=\epsilon^{N-(2 p /(p-1))}\left[K \int_{R^{N}}(w-1)_{+}^{p}+d_{w} F\left(\boldsymbol{P}^{0}\right) \epsilon^{N-2}+o\left(\epsilon^{N-2}\right)\right] .
$$

It is not hard to see that

$$
M_{*}^{(N)}=\int_{R^{N}}(w-1)_{+}^{p^{*}}
$$

Set

$$
u^{M}=\epsilon^{-2 /(p-1)}\left(u_{\epsilon}-1\right) .
$$

Let us now prove that $u^{M}$ satisfy the properties of Theorems 1.1 and 1.3.

Properties (i), (ii) and (iii) of Theorem 1.1 follow directly from those of $u_{\epsilon}$ and Lemma 6.2.

To prove Theorem 1.3, we note that the relation between $M$ and $\epsilon$ is given by

$$
M=\epsilon^{N-(2 p /(p-1))}\left\{K \int_{R^{N}}(w-1)_{+}^{p}+O\left(\epsilon^{N-2}\right)\right\} .
$$

Since $-\mu^{M}=\epsilon^{-2 /(p-1)}$, we obtain

$$
M\left(-\mu^{M}\right)^{(2 p-N(p-1)) / 2}=M \epsilon^{(2 p /(p-1))-N}=K \int_{R^{N}}(w-1)_{+}^{p}+o(1),
$$

which proves property (iii) of Theorem 1.3 with $\mu_{0}=K \int_{R^{N}}(w-1)_{+}^{p}$. Properties (i) and (ii) of Theorem 1.3 follow from the properties of $u_{\epsilon}$.

\section{Appendix A. Proof of Lemma 5.3}

In this appendix, we prove the technical lemma (Lemma 5.3). The key is to expand $\Phi_{\epsilon, \boldsymbol{P}}$ and $\partial_{k}^{l} \Phi_{\epsilon, \boldsymbol{P}}$.

Recall that $\tilde{\Phi}_{\epsilon, \boldsymbol{P}}^{0}$ is defined by (3.8). We first need the following lemma.

Lemma A 1. Let $\tilde{\Phi}_{\epsilon, \boldsymbol{P}}$ be defined by (3.8). We then have that $\Phi_{\epsilon, \boldsymbol{P}}$ is $C^{1}$ in $\boldsymbol{P}$. Moreover, we have

$$
\Phi_{\epsilon, \boldsymbol{P}}=\epsilon^{N-2} \tilde{\Phi}_{\epsilon, \boldsymbol{P}}^{0}+o\left(\epsilon^{N-2}\right)
$$

and

$$
\partial_{i}^{j} \Phi_{\epsilon, \boldsymbol{P}}=\epsilon^{N-2} \partial_{i}^{j} \tilde{\Phi}_{\epsilon, \boldsymbol{P}}^{0}+O\left(\epsilon^{N-2}\right) .
$$


Proof. Equation (A 1) has been proved in Lemma 3.4. To prove (A 2), we note that

$$
\tilde{\Phi}_{\epsilon, \boldsymbol{P}}^{0}=\Phi_{\epsilon, \boldsymbol{P}}^{0}+O\left(\epsilon^{N-2}\right) .
$$

Put

$$
\Psi_{\epsilon}:=\partial_{i}^{j} \Phi_{\epsilon, \boldsymbol{P}}-\epsilon^{N-2} \partial_{i}^{j} \tilde{\Phi}_{\epsilon, \boldsymbol{P}}^{0},
$$

and we decompose $\Psi_{\epsilon}$ into two parts

$$
\Psi_{\epsilon}=\sum_{k, l} \tilde{\alpha}_{i k}^{j l} \partial_{k}^{l} w_{\epsilon, \boldsymbol{P}}+\Psi_{\epsilon}^{\perp}, \Psi_{\epsilon}^{\perp} \in \mathcal{K}_{\epsilon, \boldsymbol{P}}^{\perp}
$$

where $\tilde{\alpha}_{i k}^{j l}$ are some constants.

We will show that

$$
\tilde{\alpha}_{i k}^{j l}=O\left(\epsilon^{N-1}\right)
$$

and

$$
\left\|\Psi_{\epsilon}^{\perp}\right\|_{t, 2, \beta}=O\left(\epsilon^{N-2}\right) .
$$

We first note that, since $\Phi_{\epsilon, \boldsymbol{P}} \perp \mathcal{K}_{\epsilon, \boldsymbol{P}}$,

$$
\epsilon^{-N} \int_{\Omega} \frac{\partial \Phi_{\epsilon, \boldsymbol{P}}}{\partial P_{i}^{j}} \frac{\partial w_{\epsilon, \boldsymbol{P}}}{\partial P_{k}^{l}}=-\epsilon^{-N} \int_{\Omega} \Phi_{\epsilon, \boldsymbol{P}} \frac{\partial^{2} w_{\epsilon, \boldsymbol{P}}}{\partial P_{i}^{j} \partial P_{k}^{l}}
$$

Hence,

$$
\begin{aligned}
\sum_{k, l} \tilde{\alpha}_{i k}^{j l} \epsilon^{-N} \int_{\Omega} \frac{\partial w_{\epsilon, \boldsymbol{P}}}{\partial P_{k}^{l}} \frac{\partial w_{\epsilon, \boldsymbol{P}}}{\partial P_{k^{\prime}}^{l^{\prime}}} \\
=-\epsilon^{N-2} \epsilon^{-N} \int_{\Omega} \Phi_{\epsilon, \boldsymbol{P}} \partial_{i}^{j} \partial_{k^{\prime}}^{l^{\prime}} w_{\epsilon, \boldsymbol{P}}+\epsilon^{-N} \int_{\Omega} \epsilon^{N-2} \Phi_{\epsilon, \boldsymbol{P}}^{0} \partial_{i}^{j} \partial_{k^{\prime}}^{l^{\prime}} w_{\epsilon, \boldsymbol{P}}+O\left(\epsilon^{N-3}\right) \\
=O\left(\epsilon^{N-3}\right) .
\end{aligned}
$$

Since

$$
\epsilon^{-N} \int_{\Omega} \frac{\partial w_{\epsilon, \boldsymbol{P}}}{\partial P_{k}^{l}} \frac{\partial w_{\epsilon, \boldsymbol{P}}}{\partial P_{k^{\prime}}^{l^{\prime}}}=\epsilon^{-2}(\Gamma+o(1)) \delta_{k k^{\prime}} \delta_{l l^{\prime}},
$$

where $\Gamma>0$, we obtain $\tilde{\alpha}_{i k}^{j l}=O\left(\epsilon^{N-1}\right)$, which proves (A 4).

Next we observe that

$$
S_{\epsilon}\left(w_{\epsilon, \boldsymbol{P}}+\Phi_{\epsilon, \boldsymbol{P}}\right)=\sum_{i j} \beta_{i}^{j}(P) \frac{\partial w_{\epsilon, \boldsymbol{P}}}{\partial P_{i}^{j}}
$$

where $\beta_{i}^{j}(\boldsymbol{P}) \in C^{1}$.

Differentiating the above equation by $\partial / \partial P_{i}^{j}$, we have

$$
S_{\epsilon}^{\prime}\left(w_{\epsilon, \boldsymbol{P}}+\Phi_{\epsilon, \boldsymbol{P}}\right)\left(\frac{\partial w_{\epsilon, \boldsymbol{P}}}{\partial P_{i}^{j}}+\frac{\partial \Phi_{\epsilon, \boldsymbol{P}}}{\partial P_{i}^{j}}\right)-\sum_{k, l} \beta_{k}^{l}(P) \frac{\partial^{2} w_{\epsilon, \boldsymbol{P}}}{\partial P_{i}^{j} \partial P_{k}^{l}} \in \mathcal{C}_{\epsilon, \boldsymbol{P}}
$$


At a critical point $\boldsymbol{P}=\boldsymbol{Q}_{\epsilon}$ of $K_{\epsilon}(\boldsymbol{P})$, we must have

$$
\beta_{k}^{l}\left(\boldsymbol{Q}_{\epsilon}\right)=0, \quad k=1, \ldots, N, \quad l=1, \ldots, K .
$$

Hence, at $\boldsymbol{P}=\boldsymbol{Q}_{\epsilon}$,

$$
S_{\epsilon}^{\prime}\left(w_{\epsilon, \boldsymbol{P}}+\Phi_{\epsilon, \boldsymbol{P}}\right)\left(\Psi_{\epsilon, \boldsymbol{P}}^{\perp}\right)+S_{\epsilon}^{\prime}\left(w_{\epsilon, \boldsymbol{P}}+\Phi_{\epsilon, \boldsymbol{P}}\right)\left(\frac{\partial\left(w_{\epsilon, \boldsymbol{P}}+\epsilon^{N-2} \Phi_{\epsilon, \boldsymbol{P}}^{0}\right)}{\partial P_{i}^{j}}+\sum_{k, l} \tilde{\alpha}_{i k}^{j l} \frac{\partial w_{\epsilon, \boldsymbol{P}}}{\partial P_{k}^{l}}\right) \in \mathcal{C}_{\epsilon, \boldsymbol{P}}
$$

We now need to compute

$$
E_{\epsilon}:=S_{\epsilon}^{\prime}\left(w_{\epsilon, \boldsymbol{P}}+\Phi_{\epsilon, \boldsymbol{P}}\right)\left(\left.\frac{\partial\left(w_{\epsilon, \boldsymbol{P}}+\epsilon^{N-2} \Phi_{\epsilon, \boldsymbol{P}}^{0}\right)}{\partial P_{i}^{j}}\right|_{\boldsymbol{P}=\boldsymbol{Q}_{\epsilon}}\right) .
$$

By using Lemma 3.4, a simple computation shows that

$$
\begin{aligned}
E_{\epsilon} & =\left.\partial_{i}^{j}\right|_{\boldsymbol{P}=\boldsymbol{Q}_{\epsilon}} S_{\epsilon}\left(w_{\epsilon, \boldsymbol{P}}+\epsilon^{N-2} \Phi_{\epsilon, \boldsymbol{P}}^{0}\right) \\
& =O\left(\epsilon^{N-2}\right) .
\end{aligned}
$$

On the other hand, by the same proof as that of Proposition 2.5, it is easy to see that

$$
\Pi_{\epsilon, \boldsymbol{P}}^{\perp} \circ S_{\epsilon}^{\prime}\left(w_{\epsilon, \boldsymbol{P}}+\Phi_{\epsilon, \boldsymbol{P}}\right): \mathcal{K}_{\epsilon, \boldsymbol{P}}^{\perp} \rightarrow \mathcal{C}_{\epsilon, \boldsymbol{P}}^{\perp}
$$

is invertible for $\epsilon$ sufficiently small. Hence (since $\Psi_{\epsilon, \boldsymbol{P}}^{\perp} \in \mathcal{K}_{\epsilon, \boldsymbol{P}}^{\perp}$ ),

$$
\begin{aligned}
\left\|\Psi_{\epsilon, \boldsymbol{P}}^{\perp}\right\|_{t, 2, \beta} & \leqslant C\left\|\Pi_{\epsilon, \boldsymbol{P}}^{\perp} \circ E_{\epsilon}\right\|_{t, 2+\beta}+O\left(\epsilon^{N-2}\right) \\
& \leqslant C \epsilon^{N-2} .
\end{aligned}
$$

Equation (A 2) is thus proved.

By using Lemma A 1, we obtain the following proof.

Proof of Lemma 5.3. In order to have a unified approach that works for all dimensions $N \geqslant 3$, we need to modify $w_{\epsilon, P}$. We introduce the following notation:

$$
\begin{gathered}
\bar{w}_{\epsilon, P^{j}}=w_{\epsilon, P^{j}}+k_{w} F_{j}\left(\boldsymbol{P}, P^{j}\right)\left(\Phi_{0}\left(\frac{x-P^{j}}{\epsilon}\right)-C_{0}(N-2)\left|S^{N-1}\right| \epsilon^{N-2} H\left(x, P^{j}\right)\right), \\
\bar{w}_{\epsilon, \boldsymbol{P}}=\sum_{j=1}^{K} \bar{w}_{\epsilon, P^{j}} \\
\bar{w}_{\epsilon, \boldsymbol{P}}+\bar{\Phi}_{\epsilon, \boldsymbol{P}}=w_{\epsilon, \boldsymbol{P}}+\Phi_{\epsilon, \boldsymbol{P}} .
\end{gathered}
$$

Then, by Lemma A 1, we have

$$
\bar{\Phi}_{\epsilon, \boldsymbol{P}}=O\left(\epsilon^{N-2+\delta_{0}}\right), \quad \partial_{k}^{l} \bar{\Phi}_{\epsilon, \boldsymbol{P}}=O\left(\epsilon^{N-2}\right) .
$$


Thus we obtain

$$
\begin{aligned}
\left.\frac{\partial^{2} K_{\epsilon}(\boldsymbol{P})}{\partial P_{i}^{j} \partial P_{k}^{l}}\right|_{\boldsymbol{P}=\boldsymbol{Q}_{\epsilon}} & \\
= & \left.\left\langle\partial_{i}^{j}\left(\bar{w}_{\epsilon, \boldsymbol{P}}+\bar{\Phi}_{\epsilon, \boldsymbol{P}}\right), \partial_{k}^{l}\left(\bar{w}_{\epsilon, \boldsymbol{P}}+\bar{\Phi}_{\epsilon, \boldsymbol{P}}\right)\right\rangle_{\epsilon}\right|_{\boldsymbol{P}=\boldsymbol{Q}_{\epsilon}} \\
& +\left.\left\langle\bar{w}_{\epsilon, \boldsymbol{P}}+\bar{\Phi}_{\epsilon, \boldsymbol{P}}, \partial_{i}^{j} \partial_{k}^{l}\left(\bar{w}_{\epsilon, \boldsymbol{P}}+\bar{\Phi}_{\epsilon, \boldsymbol{P}}\right)\right\rangle_{\epsilon}\right|_{\boldsymbol{P}=\boldsymbol{Q}_{\epsilon}} \\
& -\left.\left.\epsilon^{-N} \int_{\Omega} p\left(\bar{w}_{\epsilon, \boldsymbol{Q}_{\epsilon}}+\bar{\Phi}_{\epsilon, \boldsymbol{Q}_{\epsilon}}-1\right)_{+}^{p-1} \partial_{i}^{j}\left(\bar{w}_{\epsilon, \boldsymbol{P}}+\bar{\Phi}_{\epsilon, \boldsymbol{P}}\right)\right|_{\boldsymbol{P}=\boldsymbol{Q}_{\epsilon}} \partial_{k}^{l}\left(\bar{w}_{\epsilon, \boldsymbol{P}}+\bar{\Phi}_{\epsilon, \boldsymbol{P}}\right)\right|_{\boldsymbol{P}=\boldsymbol{Q}_{\epsilon}} \\
& -\left.\epsilon^{-N} \int_{\Omega}\left(\bar{w}_{\epsilon, Q_{\epsilon}}+\bar{\Phi}_{\epsilon, Q_{\epsilon}}-1\right)_{+}^{p} \partial_{k}^{l} \partial_{i}^{j}\left(\bar{w}_{\epsilon, \boldsymbol{P}}+\bar{\Phi}_{\epsilon, P}\right)\right|_{\boldsymbol{P}=\boldsymbol{Q}_{\epsilon}}
\end{aligned}
$$

(since $\boldsymbol{P}=\boldsymbol{Q}_{\epsilon}$ is a critical point)

$$
\begin{aligned}
=\langle & \left.\partial_{i}^{j}\left(\bar{w}_{\epsilon, \boldsymbol{P}}+\bar{\Phi}_{\epsilon, \boldsymbol{P}}\right), \partial_{k}^{l}\left(\bar{w}_{\epsilon, \boldsymbol{P}}+\bar{\Phi}_{\epsilon, \boldsymbol{P}}\right)\right\rangle\left._{\epsilon}\right|_{\boldsymbol{P}=\boldsymbol{Q}_{\epsilon}} \\
& -\left.\left.\epsilon^{-N} \int_{\Omega} p\left(\bar{w}_{\epsilon, \boldsymbol{Q}_{\epsilon}}+\bar{\Phi}_{\epsilon, \boldsymbol{Q}_{\epsilon}}-1\right)_{+}^{p-1} \partial_{i}^{j}\left(\bar{w}_{\epsilon, \boldsymbol{P}}+\bar{\Phi}_{\epsilon, \boldsymbol{P}}\right)\right|_{\boldsymbol{P}=\boldsymbol{Q}_{\epsilon}} \partial_{k}^{l}\left(\bar{w}_{\epsilon, \boldsymbol{P}}+\bar{\Phi}_{\epsilon, \boldsymbol{P}}\right)\right|_{\boldsymbol{P}=\boldsymbol{Q}_{\epsilon}} \\
= & \left.\partial_{k}^{l}\left(\bar{w}_{\epsilon, \boldsymbol{P}}\right), \partial_{i}^{j}\left(\bar{w}_{\epsilon, \boldsymbol{P}}\right)\right\rangle\left._{\epsilon}\right|_{\boldsymbol{P}=\boldsymbol{Q}_{\epsilon}} \\
& -\left.\left.\epsilon^{-N} \int_{\Omega} p\left(\bar{w}_{\epsilon, \boldsymbol{Q}_{\epsilon}}+\bar{\Phi}_{\epsilon, \boldsymbol{Q}_{\epsilon}}-1\right)_{+}^{p-1} \partial_{i}^{j}\left(\bar{w}_{\epsilon, \boldsymbol{P}}\right)\right|_{\boldsymbol{P}=\boldsymbol{Q}_{\epsilon}} \partial_{k}^{l}\left(\bar{w}_{\epsilon, \boldsymbol{P}}\right)\right|_{\boldsymbol{P}=\boldsymbol{Q}_{\epsilon}} \\
+ & \left.\left\langle\partial_{k}^{l}\left(\bar{w}_{\epsilon, \boldsymbol{P}}\right), \partial_{i}^{j}\left(\bar{\Phi}_{\epsilon, \boldsymbol{P}}\right)\right\rangle_{\epsilon}\right|_{\boldsymbol{P}=\boldsymbol{Q}_{\epsilon}} \\
& -\left.\left.\epsilon^{-N} \int_{\Omega} p\left(\bar{w}_{\epsilon, \boldsymbol{Q}_{\epsilon}}+\bar{\Phi}_{\epsilon, \boldsymbol{Q}_{\epsilon}}-1\right)_{+}^{p-1} \partial_{i}^{j}\left(\bar{\Phi}_{\epsilon, \boldsymbol{P}}\right)\right|_{\boldsymbol{P}=\boldsymbol{Q}_{\epsilon}} \partial_{k}^{l}\left(\bar{w}_{\epsilon, \boldsymbol{P}}\right)\right|_{\boldsymbol{P}=\boldsymbol{Q}_{\epsilon}} \\
+ & \left.\left\langle\partial_{k}^{l}\left(\bar{\Phi}_{\epsilon, \boldsymbol{P}}\right), \partial_{i}^{j}\left(\bar{w}_{\epsilon, \boldsymbol{P}}\right)\right\rangle_{\epsilon}\right|_{\boldsymbol{P}=\boldsymbol{Q}_{\epsilon}} \\
& -\left.\left.\epsilon^{-N} \int_{\Omega} p\left(\bar{w}_{\epsilon, \boldsymbol{Q}_{\epsilon}}+\bar{\Phi}_{\epsilon, \boldsymbol{Q}_{\epsilon}}-1\right)_{+}^{p-1} \partial_{i}^{j}\left(\bar{w}_{\epsilon, \boldsymbol{P}}\right)\right|_{\boldsymbol{P}=\boldsymbol{Q}_{\epsilon}} \partial_{k}^{l}\left(\bar{\Phi}_{\epsilon, \boldsymbol{P}}\right)\right|_{\boldsymbol{P}=\boldsymbol{Q}_{\epsilon}} \\
+ & \left.\left\langle\partial_{k}^{l}\left(\bar{\Phi}_{\epsilon, \boldsymbol{P}}\right), \partial_{i}^{j}\left(\bar{\Phi}_{\epsilon, \boldsymbol{P}}\right)\right\rangle_{\epsilon}\right|_{\boldsymbol{P}=\boldsymbol{Q}_{\epsilon}} \\
& -\left.\left.\epsilon^{-N} \int_{\Omega} p\left(\bar{w}_{\epsilon, \boldsymbol{Q}_{\epsilon}}+\bar{\Phi}_{\epsilon, \boldsymbol{Q}_{\epsilon}}-1\right)_{+}^{p-1} \partial_{i}^{j}\left(\bar{\Phi}_{\epsilon, \boldsymbol{P}}\right)\right|_{\boldsymbol{P}=\boldsymbol{Q}_{\epsilon}} \partial_{k}^{l}\left(\bar{\Phi}_{\epsilon, \boldsymbol{P}}\right)\right|_{\boldsymbol{P}=\boldsymbol{Q}_{\epsilon}} \\
= & I_{1}+I_{2}+I_{3}+I_{4},
\end{aligned}
$$

where $I_{i}, i=1,2,3,4$, are defined in the last equality.

By (A 6),

$$
\begin{aligned}
I_{4} & =o\left(\epsilon^{N-2}\right), \\
I_{2} & =\int_{\Omega_{\epsilon, P^{j}}} S_{\epsilon}^{\prime}\left(\boldsymbol{w}_{\epsilon, \boldsymbol{Q}_{\epsilon}}\right)\left(\left.\partial_{i}^{j}\right|_{\boldsymbol{P}=\boldsymbol{Q}_{\epsilon}} \bar{w}_{\epsilon, \boldsymbol{P}}\right)\left(\left.\partial_{k}^{l}\right|_{\boldsymbol{P}=\boldsymbol{Q}_{\epsilon}} \bar{\Phi}_{\epsilon, \boldsymbol{P}}\right) \\
& =o\left(\epsilon^{N-2}\right) .
\end{aligned}
$$

Similarly, we have

$$
I_{3}=o\left(\epsilon^{N-2}\right)
$$


It remains to compute $I_{1}$. The computation of $I_{1}$ is as follows

$$
\begin{aligned}
I_{1} & =\left.\left.\int_{\Omega_{\epsilon}} S_{\epsilon}^{\prime}\left(\bar{w}_{\epsilon, \boldsymbol{Q}_{\epsilon}}\right) \partial_{i}^{j}\right|_{\boldsymbol{P}=\boldsymbol{Q}_{\epsilon}} \bar{w}_{\epsilon, \boldsymbol{P}} \partial_{k}^{l}\right|_{\boldsymbol{P}=\boldsymbol{Q}_{\epsilon}} \bar{w}_{\epsilon, \boldsymbol{P}} \\
& =\left.\int_{\Omega_{\epsilon}} \partial_{i}^{j}\left[S_{\epsilon}\left(\bar{w}_{\epsilon, \boldsymbol{P}}\right) \partial_{k}^{l} \bar{w}_{\epsilon, \boldsymbol{P}}\right]\right|_{\boldsymbol{P}=\boldsymbol{Q}_{\epsilon}} \\
& =\left.\partial_{i}^{j} \partial_{k}^{l} J_{\epsilon}\left(\bar{w}_{\epsilon, \boldsymbol{P}}\right)\right|_{\boldsymbol{P}=\boldsymbol{Q}_{\epsilon} .} .
\end{aligned}
$$

Note that

$$
J_{\epsilon}\left(\bar{w}_{\epsilon, \boldsymbol{P}}\right)=K I(w)-c_{w} F(\boldsymbol{P}) \epsilon^{N-2}+o\left(\epsilon^{N-2}\right) .
$$

We now need to show that the above equality is true in $C^{2}$ for $\boldsymbol{P}$. Although intuitively it should be true, the proofs are quite complicated, due to the fact that when we differentiate $P^{j}$ we are also differentiating $x$, since the variables involved are $\left(x-P^{j}\right) / \epsilon$. The key is to separate these two differentiations. To this end, we introduce

$$
D_{i}^{j}:=\frac{\partial}{\partial P_{i}^{j}}+\frac{\partial}{\partial x_{i}} \text {. }
$$

We then have

$$
I_{1}=\left.\int_{\Omega_{\epsilon, P^{j}}} D_{i}^{j}\left[S_{\epsilon}\left(\bar{w}_{\epsilon, \boldsymbol{P}}\right) \partial_{k}^{l} \bar{w}_{\epsilon, \boldsymbol{P}}\right]\right|_{\boldsymbol{P}=\boldsymbol{Q}_{\epsilon}}+o\left(\epsilon^{N-2}\right) .
$$

Note that

$$
D_{i}^{j} g\left(\frac{\left|x-P^{j}\right|}{\epsilon}\right)=0
$$

for any radial function $g$.

So

$$
\begin{aligned}
S_{\epsilon}\left(\bar{w}_{\epsilon, \boldsymbol{P}}\right) & =\left(\bar{w}_{\epsilon, \boldsymbol{P}}-1\right)_{+}^{p}-\sum_{s=1}^{K}\left(w_{s}-1\right)_{+}^{p}-p k_{w} \sum_{s=1}^{K}\left(w_{s}-1\right)_{+}^{p-1} F_{s}\left(\boldsymbol{P}, P^{s}\right)\left(\Phi_{0}+1\right) \\
& =\epsilon^{N-2} p k_{w} \sum_{s=1}^{K}\left(w_{s}-1\right)_{+}^{p-1}\left(F_{s}(\boldsymbol{P}, x)-F_{s}\left(\boldsymbol{P}, P^{s}\right)\right)\left(\Phi_{0}+1\right)+o\left(\epsilon^{N-2}\right) .
\end{aligned}
$$

Thus

$$
\begin{aligned}
D_{i}^{j} & {\left[S_{\epsilon}\left(\bar{w}_{\epsilon, \boldsymbol{P}}\right) \partial_{k}^{l} \bar{w}_{\epsilon, \boldsymbol{P}}\right] } \\
& =D_{i}^{j}\left(\epsilon^{N-2} p k_{w} \sum_{s=1}^{K}\left(w_{s}-1\right)_{+}^{p-1}\left(F_{j}(\boldsymbol{P}, x)-F_{j}\left(\boldsymbol{P}, P^{s}\right)\right)\left(\Phi_{0}+1\right) \partial_{k}^{l} w_{l}\right)+o\left(\epsilon^{N-2}\right) \\
& =\epsilon^{N-2} p k_{w} \sum_{s=1}^{K}\left(w_{s}-1\right)_{+}^{p-1} D_{i}^{j}\left(F_{s}(\boldsymbol{P}, x)-F_{s}\left(\boldsymbol{P}, P^{s}\right)\right)\left(\Phi_{0}+1\right) \partial_{k}^{l} w_{l}+o\left(\epsilon^{N-2}\right) .
\end{aligned}
$$


Hence

$$
\begin{aligned}
I_{1} & =\epsilon^{N-2} p k_{w} \int_{\Omega_{\epsilon, P} l}(w-1)_{+}^{p-1} \partial_{k}^{l} \partial_{i}^{j} F(\boldsymbol{P}) y_{k} \frac{\partial w}{\partial y_{k}}\left(\Phi_{0}+1\right)+o\left(\epsilon^{N-2}\right) \\
& =-\left.c_{w} \epsilon^{N-2} \partial_{k}^{l} \partial_{i}^{j} F(\boldsymbol{P})\right|_{\boldsymbol{P}=\boldsymbol{Q}_{\epsilon}}+o\left(\epsilon^{N-2}\right) .
\end{aligned}
$$

Lemma 5.3 is thus proved.

\section{Appendix B. Proof of Lemma 2.6}

In this appendix, we prove the regularity result Lemma 2.6. Recall that $0<\beta<$ $\left(N / t^{\prime}\right)-1$.

We first prove the case when $K=1$. In this case, we rescale the domain $\Omega$ and the functions as follows:

$$
x=\epsilon y+P^{1}, \quad \tilde{\Phi}(y)=\Phi\left(\epsilon y+P^{1}\right), \quad \tilde{f}(y)=f\left(\epsilon y+P^{1}\right) .
$$

Then $\tilde{\Phi}$ satisfies

$$
\Delta \tilde{\Phi}+\tilde{f}=0, \quad \text { in } \Omega_{\epsilon, P^{1}} .
$$

We extend $\tilde{\Phi}$ and $\tilde{f}$ to $R^{N}$ by putting 0 outside $\Omega_{\epsilon, P^{1}}$.

Note that the Laplace operator $\Delta$ is an isomorphism from $W_{2, \beta}^{t}\left(R^{N}\right)$ to $L_{2+\beta}^{t}\left(R^{N}\right)$. See $[\mathbf{1 3}],[\mathbf{2 4}],[\mathbf{2 5}]$ and $[\mathbf{2 8}]$. Thus we have

$$
\|\Phi\|_{t, 0, \beta} \leqslant\|\tilde{\Phi}\|_{L_{\beta}^{t}\left(R^{N}\right)} \leqslant C\|\tilde{f}\|_{L_{2+\beta}^{t}\left(R^{N}\right)} \leqslant C\|f\|_{t, 2+\beta} .
$$

On the other hand, let

$$
\bar{\Phi}=\tilde{\Phi}(y) \chi\left(x-P^{1}\right),
$$

where $\chi(x)=1$ for $|x| \leqslant \delta$ and $\chi(x)=0$ for $|x| \geqslant 2 \delta$.

Then it is easy to see that $\bar{\Phi}$ satisfies

$$
\Delta_{y} \bar{\Phi}+\chi \tilde{f}+2 \nabla_{y} \chi \nabla \tilde{\Phi}+\Delta_{y} \chi \tilde{\Phi}=0, \bar{\Phi} \in W_{2, \beta}^{t}\left(R^{N}\right) .
$$

Hence

$$
\begin{aligned}
\|\bar{\Phi}\|_{W_{2, \beta}^{t}\left(R^{N}\right)} & \leqslant C\|f\|_{t, 2+\beta}+C\left\|\nabla_{y} \chi \nabla \tilde{\Phi}\right\|_{t, 2+\beta} \\
& \leqslant C\|f\|_{t, 2+\beta}+\delta\|\Phi\|_{t, 2, \beta}
\end{aligned}
$$

for any small $\delta$. Here we have used the interpolation inequality.

But outside $B_{\delta}\left(P^{1}\right)$, we have

$$
\|\Phi\|_{W_{2, \beta}^{t}\left(\Omega \backslash B_{\delta}\left(P^{1}\right)\right)} \leqslant C\|f\|_{t, 2+\beta} .
$$

Thus

$$
\|\Phi\|_{t, 2, \beta} \leqslant C\|f\|_{t, 2+\beta}+\delta\|\Phi\|_{t, 2+\beta} .
$$

Lemma 2.6 is thus proved for the case $K=1$. 
Next we prove the case when $K=2$. The other cases $K>2$ can be proved similarly.

We divide the domain into two parts: $\Omega_{1} \bigcup \Omega_{2}=\Omega$ and $\Omega_{1} \cap \Omega_{2}=\phi$. We choose $\chi_{1}$ and $\chi_{2}$ such that $\chi_{1}(x)=1$ for $x \in B_{\delta}\left(P^{1}\right)$ and $\chi_{1}(x)=0$ for $\in \Omega \backslash B_{\delta}\left(P^{2}\right)$. Similarly, we require that $\chi_{2}(x)=1$ for $x \in B_{\delta}\left(P^{2}\right)$ and $\chi_{2}(x)=0$ for $\in \Omega \backslash B_{\delta}\left(P^{1}\right)$. Moreover, we can assume that $\chi_{1}(x)+\chi_{2}(x)=1$ for $x \in \Omega$.

Let $\Phi^{i}, i=1,2$, be the unique solution of

$$
\epsilon^{2} \Delta \Phi^{i}+\chi_{i}(x) f=0, \quad \text { in } \Omega, \quad \Phi^{i} \in H_{0}^{1}(\Omega) .
$$

Then we have

$$
\Phi(x)=\Phi_{1}+\Phi_{2}
$$

and

$$
\left\|\Phi_{j}\right\|_{W_{2, \beta}^{t}\left(\Omega_{\epsilon, P^{j}}\right)} \leqslant C\|f\|_{t, 2+\beta}, \quad j=1,2 .
$$

Since $\beta>0$,

$$
\begin{gathered}
\left(\sigma_{\boldsymbol{P}}(x)\right)^{\beta} \leqslant\left(1+\frac{\left|x-P^{j}\right|}{\epsilon}\right)^{\beta}, \\
\left\|\Phi_{j}\right\|_{t, 2, \beta} \leqslant\|\Phi\|_{W_{2, \beta}^{t}\left(\Omega_{\epsilon, P^{j}}\right)}, \quad j=1,2 .
\end{gathered}
$$

So we have

$$
\|\Phi\|_{t, 2, \beta} \leqslant C\left\|\Phi_{1}\right\|_{t, 2, \beta}+C\left\|\Phi_{2}\right\|_{t, 2, \beta} \leqslant C\|f\|_{t, 2+\beta} .
$$

Lemma 2.6 is thus proved.

Acknowledgements. The author thanks the referee for critically reading the manuscript and for many valuable suggestions. This research is supported by an earmarked grant from RGC of Hong Kong.

\section{References}

1. A. BAhri AND J. M. Coron, On a nonlinear elliptic equation involving the critical Sobolev exponent: the effect of the topology of the domain, Commun. Pure Appl. Math. 41 (1988), 255-294.

2. A. BAHRI, Y. Li AND O. ReY, On a variational problem with lack of compactness: the topological effect of the critical points at infinity, Calc. Var. PDE 3 (1995), 67-93.

3. C. BANDle AND M. Flucher, Harmonic radius and concentration of energy; hyperbolic radius and Liouville's equations $\Delta U=\mathrm{e}^{U}$ and $\Delta U=U^{\frac{n+2}{n-2}}, S I A M$ Rev. 38 (1996), 191-238.

4. C. Bandle and M. Marcus, A priori estimates and the boundary values of solutions for a problem arising in plasma physics, Nonlinear Analysis TMA 7 (1983), 439-451.

5. S. BARAKET AND F. PACARD, Construction of singular limits for a semilinear elliptic equation in dimension 2, Calc. Var. PDE 6 (1998), 6-38.

6. P. Bates And G. Fusco, Equilibria with many nuclei for the Cahn-Hilliard equation, J. Diff. Eqns 162 (2000), 283-356.

7. P. Bates, E. N. Dancer and J. Shi, Multi-spike stationary solutions of the CahnHilliard equation in higher-dimension and instability, Adv. Diff. Eqns 4 (1999), 1-69.

8. H. BeRESTYCKI AND H. BREzIS, On a free boundary problem arising in plasma physics, Nonlinear Analysis TMA 4 (1980), 415-436. 
9. H. BeRESTYCKI AND P. L. LiOns, Nonlinear scalar field equations, I, Existence of a ground state, Arch. Ration. Mech. Analysis 82 (1983), 313-345.

10. H. Brezis and L. Nirenberg, Positive solutions of nonlinear elliptic equations involving critical Sobolev exponents, Commun. Pure Appl. Math. 36 (1983), 437-477.

11. H. BRezis AND L. A. Peletier, Asymptotics for elliptic equations involving critical growth, in Partial differential equations and the calculus of variations, vol. 1, pp. 149-192 (Birkhäuser, Boston, MA, 1989).

12. L. Caffarelli and A. Friedman, Asymptotic estimates for the plasma problem, Duke Math. J. 47 (1980), 705-742.

13. M. CAntor, Spaces of functions with asymptotic conditions on $R^{n}$, Indiana Univ. Math. J. 24 (1975), 897-902.

14. A. Chang And C.-S. Lin, in preparation.

15. M. Del Pino, P. Felmer AND J. Wei, Multiple-peaked solutions for a singularly perturbed Dirichlet problem, Calc. Var. PDE 10 (2000), 119-134.

16. N. Ghoussoub And C. Gui, Multi-peak solutions for a semilinear Neumann problem involving the critical Sobolev exponent, Math. Z. 229 (1998), 443-474.

17. C. GuI, Multi-peak solutions for a semilinear Neumann problem, Duke Math. J. 84 (1996), 739-769.

18. C. GUI AND J. WEI, Multiple interior peak solutions for some singular perturbation problems, J. Diff. Eqns 158 (1999), 1-27.

19. C. Gui, J. Wei And M. Winter, Multiple boundary peak solutions for some singularly perturbed Neumann problems, Ann. Inst. H. Poincaré Analyse Non Linéare 17 (2000), 47-82.

20. M. Flucher AND J. WeI, Asymptotic behavior of elliptic free boundary problem, Math. Z. 228 (1998), 683-703.

21. J. L. KAzDAm And F. W. WARner, Curvature functions for compact 2-manifolds, Ann. Math. 99 (1974), 14-47.

22. M. KowALCZYK, Multiple spike layers in the shadow Gierer-Meinhardt system: existence of equilibria and approximate invariant manifold, Duke Math. J. 98 (1999), 59-111.

23. Y.-Y. LI, On a singularly perturbed equation with Neumann boundary condition, Commun. Partial Diff. Eqns 23 (1998), 487-545.

24. R. B. LockHART, Fredholm properties of a class of elliptic operators on noncompact manifolds, Duke Math. J. 48 (1981), 289-312.

25. R. MCOwen, The behavior of the Laplacian on weighted Sobolev spaces, Commun. Pure Appl. Math. 32 (1979), 783-795.

26. W.-M. Ni AND I. TAKAGI, On the shape of the least energy solution to a semilinear Neumann problem, Commun. Pure Appl. Math. 41 (1991), 819-851.

27. W.-M. Ni AND I. TAKAGi, Point-condensation generated by a reaction-diffusion system in axially symmetric domains, Japan J. Indust. Appl. Math. 12 (1995), 327-365.

28. L. NiRENBERG AND H. WALKER, The nullspaces of elliptic partial differential equations in $R^{n}$, J. Math. Analysis Appl. 42 (1973), 271-301.

29. O. REY, Proof of two conjectures of H. Brezis and L. A. Peletier, Manus. Math. 65 (1989), 19-37.

30. O. REY, The role of Green's function in a nonlinear elliptic equation involving the critical Sobolev exponent, J. Funct. Analysis 89 (1990), 1-52.

31. R. TEmAM, A nonlinear eigenvalue problem; the shape at equilibrium of a confined plasma, Arch. Ration. Mech. Analysis 60 (1975), 51-73.

32. S. WANG, Some nonlinear elliptic equations with subcritical growth and critical behavior, Houston J. Math. 16 (1990), 559-572.

33. X. WANG AND J. WeI, On the equation $\Delta u+K(x) u^{\frac{n+2}{n-2} \pm \epsilon^{2}}=0$ in $R^{n}$, Rend. Circolo Matem. Palermo 48 (1995), 365-400. 
34. J. WEI, On the interior spike layer solutions for some singular perturbation problems, Proc. R. Soc. Edinb. A 128 (1998), 849-874.

35. J. WEI, Asymptotic behavior of least energy solutions to a semilinear Dirichlet problem near the critical exponent, J. Math. Soc. Japan 48 (1998), 1-52.

36. J. Wei AND M. Winter, Stationary solutions of Cahn-Hilliard equations, Ann. Inst. H. Poincaré Analyse Non Linéare 15 (1998), 459-492.

37. J. WEI AND M. Winter, Multiple boundary spike solutions for a wide class of singular perturbation problems, J. Lond. Math. Soc. (2) 59 (1999), 585-606.

38. G. WolAnsky, On the evolution of self-interacting clusters and applications to semilinear equations of exponential nonlinearity, J. Analyse Math. 59 (1992), 251-272.

39. G. Wolansky, Critical behavior of semi-linear elliptic equations with sub-critical exponents, Nonlinear Analysis TMA 26 (1996), 971-995. 